\title{
Projector augmented-wave formulation of response to strain and electric-field perturbation within density functional perturbation theory
}

\author{
A. Martin, ${ }^{1,2, *}$ M. Torrent, ${ }^{1, \dagger}$ and R. Caracas ${ }^{2,+}$ \\ ${ }^{1}$ CEA, DAM, DIF, F-91297 Arpajon, France \\ ${ }^{2}$ CNRS, ENS de Lyon, Université Claude-Bernard Lyon 1, Laboratoire de Géologie de Lyon, UMR 5276, Lyon, France
}

(Received 1 May 2018; revised manuscript received 28 November 2018; published 27 March 2019)

\begin{abstract}
A formulation of the response of a system to strain and electric field perturbations in the pseudopotential-based density functional perturbation theory has been proposed by Hamann et al. [D. R. Hamann, X. Wu, K. M. Rabe, and D. Vanderbilt, Phys. Rev. B 71, 035117 (2005)], using an elegant formalism based on the expression of density functional theory (DFT) total energy in reduced coordinates, the key quantity being the metric tensor. We extend this formulation to the projector augmented-wave approach. In this context, we express the full elastic tensor including the clamped-atom tensor, the atomic-relaxation contributions, and the response to electric field change (piezoelectric tensor and effective charges). With this, we are able to compute the elastic tensor for all materials within a fully analytical formulation. The comparison with finite difference calculations on simple systems shows excellent agreement. This formalism has been implemented in the plane-wave-based DFT ABINIT software package. We apply it to the computation of elastic properties of low-symmetry systems of geophysical interest.
\end{abstract}

DOI: 10.1103/PhysRevB.99.094112

\section{INTRODUCTION}

Traditionally, computation of elastic properties of materials, namely the elastic constant tensor (ECT), in $a b$ initio simulations based on density-functional theory (DFT), made use of linear stress-strain relations. The residual stresses are calculated under finite strain; after enough independent deformations are applied, the ECT is obtained solving a linear system of stress-strain equations. In case of highly symmetric crystals, e.g., cubic or hexagonal, these are straightforward and fast simulations. However, for lower symmetry systems, the number of necessary calculations increases rapidly. In the extreme case, for triclinic crystals, the ECT contains 21 independent values, whose determination requires at least 43 independent calculations. As the computation of stresses is done once the atomic positions are relaxed, i.e., the determination of such a triclinic ECT presents a heavy computational cost. Such low-symmetry systems are oftentimes found when computing the elasticity along solid solution joins or studying the effect of atomic impurities and lattice defects, where random configurations break the symmetry of the parent structures.

An elegant and fast alternative to the linear stress-strain relations is the determination of the ECT from a Taylor expansion of the energy with respect to infinitesimal perturbation of the strain. In this context, the ECT represents the second-order derivatives of the energy with respect to lattice deformations. A first development was proposed in the early days of density-functional perturbation theory (DFPT)

\footnotetext{
*alexandre.martin@uliege.be

†marc.torrent@cea.fr

†razvan.caracas@ens-lyon.fr
}

by Baroni et al. [1], who derived a set of equations using a Green's function approach [2]. Later, an explicit formalism was developed by Hamann et al. [3] within the DFPT, where the perturbations were strain perturbations of the lattice. The development in Taylor series was realized in the reduced crystallographic space, which allowed for universal expressions independent of the symmetry. This approach and subsequent implementation in the standard formalism of norm-conserving pseudopotentials has been successfully applied to a variety of materials.

Here we extend this perturbative approach to obtain the ECT from direct DFPT calculations realized in the projector augmented-wave (PAW) approach [4,5]. While based on a more complex formalism than the norm-conserving pseudopotentials, the PAW approach presents net advantages in terms of accuracy, matching that of all-electron calculations, and rapidity, matching or even surpassing that of normconserving calculations. The DFPT within the PAW formalism was recently developed and implemented in several DFT packages [6-8], including perturbations only due to atomic displacements and partly due to homogeneous electric field. Regarding the response to an homogeneous electric field, it should be noted that it is also possible now to compute the clamped dielectric tensor in the PAW approach [9] using the formalism of the modern theory of magnetization [10].

Our theoretical development and practical implementation in the plane-wave-based DFT ABINIT software package follow closely the work of Refs. [3,11]. Throughout our paper, we use notations consistent to Ref. [5]. Our paper is an extension and application to the PAW formalism; it specifically contains two parts: the generalization of the derivatives with respect to the electric field and the development of the derivatives with respect to strains. The paper is organized as follows. In Sec. II, we present the different theoretical formalisms 
involved in this development, including PAW-based DFPT and derivatives with respect to the strain and the electric field. In Sec. III, we detail the strain perturbation in the PAW approach. Section IV summarizes the practical implementation and its validation. We also show a few applications of the calculations on real low-symmetry systems of geophysical interest.

\section{FORMALISM}

In this first section, we briefly present the theoretical background and define the conventions and notations. For our theoretical development, we need to mix several formalisms: DFPT, PAW, metric tensor formulation, and electric field formulation of the DFT.

\section{A. Density-functional perturbation theory}

DFPT is based on the principle that, following small perturbations $\lambda_{1}, \lambda_{2}, \ldots$ applied to the system made of nuclei and electrons, it is possible to develop each physical quantity as a Taylor-series expansion:

$X\left[\lambda_{1}, \lambda_{2}, \ldots\right]=X^{(0)}+\lambda_{1} X^{\left(\lambda_{1}\right)}+\lambda_{2} X^{\left(\lambda_{2}\right)}+\lambda_{1} \lambda_{2} X^{\left(\lambda_{1} \lambda_{2}\right)}+\ldots$

In this generic expression, $X$ can be any scalar or vector quantity: energy, wave function, potential, etc., and $X^{\left(\lambda_{i}, \lambda_{j}, \ldots\right)}$ is proportional to the derivative of $\mathrm{X}$ with respect to $\lambda_{i}, \lambda_{j}, \ldots$ In the remainder of this paper, we focus on the first- and second-order derivatives with respect to atomic displacements, strains, and electric fields. A derivative with respect to the displacement of atom $\kappa$ in the direction $k$ will be noted $X^{\left(\tau_{k k}\right)}$, a derivative with respect to the strain $\epsilon$ in the directions $\alpha$ and $\beta$ will be noted $X^{(\alpha \beta)}$, a derivative with respect to an electric field $\mathcal{E}$ along the direction $j$ will be noted $X^{\left(\mathcal{E}_{j}\right)} \cdot \psi_{n}^{(\lambda)}$ is the derivative of the wave function with respect to the perturbation $\lambda$.

In the previous literature, the notations used to express the total and partial derivatives vary from one paper to another. Hereafter, we use the $\frac{d}{d \lambda}$ symbol for a total derivative selfconsistently determined, a partial derivative symbol $\left.\frac{\partial}{\partial \lambda}\right|_{\psi^{(0)}}$ for a derivative that does not depend on the change of wave functions, and the $\delta$ symbol is the functional derivative. These derivatives are connected as follows:

$$
\frac{d \mathcal{H}}{d \lambda}=\left.\frac{\partial \mathcal{H}}{d \partial}\right|_{\psi^{(0)}}+\int_{\mathbb{R}^{3}} \frac{\delta^{2} E_{H x c}[\rho]}{\delta \rho(\mathbf{r}) \delta \rho\left(\mathbf{r}^{\prime}\right)} \rho^{(\lambda)}\left(\mathbf{r}^{\prime}\right) d \mathbf{r}^{\prime} .
$$

In the DFPT as formulated by Gonze and Vigneron [12], the higher order derivatives of the energy are obtained from applying the perturbations on the variational problem. The extension of this problem is described with the $2 N+1$ theorem [13]. It is possible, with this theorem, to obtain the $2 N$ th and the $(2 N+1)$ th order derivatives of the energy only from the derivatives of the wave functions up to the $N$ th order [13] using, respectively, a variational and a nonvariational expression.

The extension to the second order of the variational problem for a specific perturbation $\lambda_{1}$ is given by [13]

$$
\begin{aligned}
E^{\left(\lambda_{1} \lambda_{1}\right)}\left\{\psi^{(0)} ; \psi^{\left(\lambda_{1}\right)}\right\}= & \min _{\psi^{\left(\lambda_{1}\right)}}\left\{\sum _ { n } f _ { n } \left[\left\langle\psi_{n}^{(0)}\left|\left(T+V_{\mathrm{ext}}\right)^{\left(\lambda_{1} \lambda_{1}\right)}\right| \psi_{n}^{(0)}\right\rangle+\left\langle\psi_{n}^{\left(\lambda_{1}\right)}\left|T+V_{\mathrm{ext}}-\epsilon_{n}\right| \psi_{n}^{\left(\lambda_{1}\right)}\right\rangle\right.\right. \\
& \left.+\left\langle\psi_{n}^{\left(\lambda_{1}\right)}\left|\left(T+V_{\mathrm{ext}}\right)^{\left(\lambda_{1}\right)}\right| \psi_{n}^{(0)}\right\rangle+\left\langle\psi_{n}^{(0)}\left|\left(T+V_{\mathrm{ext}}\right)^{\left(\lambda_{1}\right)}\right| \psi_{n}^{\left(\lambda_{1}\right)}\right\rangle\right]+\left.\frac{1}{2} \frac{\partial^{2} E_{H x c}[\rho]}{\partial \lambda_{1}^{2}}\right|_{\psi^{(0)}} \\
& +\left.\int_{\mathbb{R}^{3}} \frac{\partial}{\partial \lambda_{1}} \frac{\delta E_{H x c}[\rho]}{\delta \rho(\mathbf{r})}\right|_{\psi^{(0)}} \rho^{\left(\lambda_{1}\right)}(\mathbf{r}) d \mathbf{r}+\int_{\mathbb{R}^{3}} \int_{\mathbb{R}^{3}} \frac{1}{2} \frac{\delta^{2} E_{H x c}[\rho]}{\delta \rho(\mathbf{r}) \delta \rho\left(\mathbf{r}^{\prime}\right)} \rho^{\left(\lambda_{1}\right)}(\mathbf{r}) \rho^{\left(\lambda_{1}\right)} \mathbf{r}^{\prime} d \mathbf{r} d \mathbf{r}^{\prime} \\
& \left.-\sum_{n, n^{\prime}} \Lambda_{n, n^{\prime}}\left(\left\langle\psi_{n}^{\left(\lambda_{1}\right)} \mid \psi_{n^{\prime}}^{(0)}\right\rangle+\left\langle\psi_{n}^{(0)} \mid \psi_{n^{\prime}}^{\left(\lambda_{1}\right)}\right\rangle\right)\right\}
\end{aligned}
$$

where $f_{n}$ is the electronic occupancy of the band with eigenvalue $\epsilon_{n}, T$ is the kinetic operator, $V_{\text {ext }}$ is the potential external to the electronic system that includes the one created by nuclei, $E_{H x c}$ is the Hartree and exchange-correlation energy functional of the electronic density $\rho$, and $\Lambda_{n, n^{\prime}}$ are the Lagrange multipliers corresponding to the orthogonality constraints. Solving this equation yields the first-order derivative of the wave function $\psi_{n}^{\left(\lambda_{1}\right)}$, via the Sternheimer equation [14]:

$$
P_{c}\left(\mathcal{H}^{(0)}-\epsilon_{n}^{(0)}\right) P_{c}\left|\psi_{n}^{\left(\lambda_{1}\right)}\right\rangle=-P_{c} \mathcal{H}^{\left(\lambda_{1}\right)}\left|\psi_{n}^{(0)}\right\rangle,
$$

where $\mathcal{H}$ is the Hamiltonian, and $P_{c}$ are projectors onto occupied states that ensure orthogonality between $\left|\psi_{n}^{(0)}\right\rangle$ and $\left|\psi_{n}^{\left(\lambda_{1}\right)}\right\rangle$. Thanks to the projectors, we can obtain the variational expression of the second-order derivative of the energy, $E_{\text {var }}^{\left(\lambda_{1} \lambda_{1}\right)}\left\{\psi^{(0)} ; \psi^{\left(\lambda_{1}\right)}\right\}$ (Eq. (91) in Ref. [14]).

From the first-order derivative of the wave function $\psi_{n}^{\left(\lambda_{1}\right)}$, it is possible to compute second-order mixed derivatives of the energy using the nonvariational expression [15]:

$$
\begin{aligned}
& E_{\text {nonvar }}^{\left(\lambda_{1} \lambda_{2}\right)}\left\{\psi^{(0)} ; \psi^{\left(\lambda_{1}\right)}\right\} \\
& =\sum_{n} f_{n}\left[\left\langle\psi_{n}^{(0)}\left|\left(T+V_{\text {ext }}\right)^{\left(\lambda_{1} \lambda_{2}\right)}\right| \psi_{n}^{(0)}\right\rangle\right. \\
& \left.\quad+\left\langle\psi_{n}^{(0)}\left|\left(T+V_{\text {ext }}\right)^{\left(\lambda_{2}\right)}\right| \psi_{n}^{\left(\lambda_{1}\right)}\right\rangle\right]+\left.\frac{1}{2} \frac{\partial^{2} E_{H x c}[\rho]}{\partial \lambda_{1} \partial \lambda_{2}}\right|_{\psi^{(0)}} \\
& \quad+\left.\frac{1}{2} \int_{\mathbb{R}^{3}} \frac{\partial}{\partial \lambda_{2}} \frac{\delta E_{H x c}[\rho]}{\delta \rho(\mathbf{r})}\right|_{\psi^{(0)}} \rho^{\left(\lambda_{1}\right)}(\mathbf{r}) d \mathbf{r}
\end{aligned}
$$

\section{B. The projector augmented-wave method}

The widely used PAW method [4] is an extension of the pseudopotential approach combined with augmented wave techniques. The interactions between the valence electrons 
and the ions in the frozen-core approximation are represented by a pseudopotential. The variations and the nodes of the wave functions close to the cores are correctly represented using a local basis. We use the notations and conventions of Ref. [5]; in the following, we only recall the necessary quantities.

\section{The PAW transformation}

The exact wave functions are connected to the pseudowave-function via a linear transformation, expressed as a sum of atom-dependent contributions:

$$
\left|\psi_{n}\right\rangle=\sum_{i}\left(\left|\phi_{i}\right\rangle-\left|\tilde{\phi}_{i}\right\rangle\right)\left\langle\tilde{p}_{i} \mid \tilde{\psi}_{n}\right\rangle,
$$

where $i$ runs over the atom positions and angular momenta. The partial waves $\phi_{i}$ form a basis of atomic orbitals, and the $\tilde{\phi}_{i}$ are "pseudized" partial waves obtained from $\phi_{i}$ 's, without any condition for norm conservation. The $\tilde{p}_{i}$ are projectors, which are dual functions of the $\tilde{\phi}_{i}$.

Equation (6) allows us to write the total charge density as

$$
\rho(\mathbf{r})=\tilde{\rho}(\mathbf{r})+\rho_{1}(\mathbf{r})-\tilde{\rho}_{1}(\mathbf{r})+\rho_{c}(\mathbf{r}),
$$

where $\rho_{c}(\mathbf{r})$ is the frozen-core density, $\tilde{\rho}(\mathbf{r})$ is the pseudovalence-density in the plane-wave representation, and $\rho_{1}$ and $\tilde{\rho}_{1}$ are on-site densities:

$$
\begin{gathered}
\tilde{\rho}(\mathbf{r})=\sum_{n} f_{n}\left|\tilde{\psi}_{n}(\mathbf{r})\right|^{2}, \\
\rho_{1}(\mathbf{r})=\sum_{i j} \rho_{i j} \phi_{i}(\mathbf{r}) \phi_{j}(\mathbf{r}),
\end{gathered}
$$

and

$$
\tilde{\rho}_{1}(\mathbf{r})=\sum_{i j} \rho_{i j} \tilde{\phi}_{i}(\mathbf{r}) \tilde{\phi}_{j}(\mathbf{r}) .
$$

In the above expressions, the $\rho_{i j}$ scalars are the occupancies of each $i, j$ channel, defined as

$$
\rho_{i j}=\sum_{n} f_{n}\left\langle\tilde{\psi}_{n} \mid \tilde{p}_{i}\right\rangle\left\langle\tilde{p}_{j} \mid \tilde{\psi}_{n}\right\rangle
$$

As PAW uses an ultrasoft formalism, the asymptotic behavior of the Hartree potential is not preserved at long range. We introduce a compensation charge density to restore it:

$$
\hat{\rho}(\mathbf{r})=\sum_{i j l m} \rho_{i j} Q_{i j}^{l m}(\mathbf{r})
$$

where

$$
Q_{i j}^{l m}(\mathbf{r})=q_{i j}^{l} g_{l}\left(\left|\mathbf{r}-\boldsymbol{\tau}_{\kappa}\right|\right) Y_{l m}\left(\widehat{\mathbf{r}-\boldsymbol{\tau}_{\kappa}}\right) .
$$

$q_{i j}^{l}$ are the moments of the difference between the norms of the partial and the pseudopartial waves, $g_{l}$ is a shape function localized in the augmentation region, $\boldsymbol{\tau}_{\kappa}$ is the position of atom $\kappa$ associated to channels $i$ and $j . Y_{l m}$ are (real) spherical harmonics.

\section{The PAW Hamiltonian}

From the decomposition of the density [Eq. (7)], we can obtain an expression for the total energy (see Ref. [4] for the detailed description of the different terms):

$$
E=\tilde{E}+E^{1}-\tilde{E}^{1} .
$$

By differentiation of this energy with respect to the pseudo-density-operator, the pseudo-PAW-Hamiltonian becomes (with the simplified notations of Audouze et al. [11])

$$
\tilde{\mathcal{H}}=T+V_{H x c}\left[\tilde{\rho}+\hat{\rho} ; \tilde{\rho}_{c}\right]+V_{H}\left[\tilde{\rho}_{Z c}\right]+\sum_{i j}\left(\left|\tilde{p}_{i}\right\rangle D_{i j}\left\langle\tilde{p}_{j}\right|\right),
$$

where $V_{H x c}\left[\tilde{\rho}+\hat{\rho} ; \tilde{\rho}_{c}\right]$ is the sum of the Hartree potential $V_{H}[\tilde{\rho}+\hat{\rho}]$ and of the exchange-correlation potential $V_{x c}[\tilde{\rho}+$ $\left.\tilde{\rho}_{c}\right] ; V_{H}\left[\tilde{\rho}_{Z c}\right]$ is the local pseudopotential and $\tilde{\rho}_{Z c}$ is the pseudo-core-density.

The $D_{i j}$ scalars involved in the nonlocal operator are selfconsistently determined from the wave functions as

$$
D_{i j}=\hat{D}_{i j}+D_{i j}^{1}-\tilde{D}_{i j}^{1} .
$$

They are made of (i) a contribution from the compensation charge density,

$$
\hat{D}_{i j}=\frac{\partial \tilde{E}}{\partial \rho_{i j}}=\sum_{L} \int_{\mathbb{R}^{3}} \tilde{V}_{\mathrm{eff}}(\mathbf{r}) Q_{i j}^{L}(\mathbf{r}) d \mathbf{r},
$$

with

$$
\tilde{V}_{\text {eff }}=V_{H}[\tilde{\rho}+\hat{\rho}]+V_{H}\left[\tilde{\rho}_{Z c}\right]+V_{x c}\left[\tilde{\rho}+\hat{\rho} ; \tilde{\rho}_{c}\right] ;
$$

(ii) an all-electron on-site contribution:

$$
D_{i j}^{1}=\frac{\partial E^{1}}{\partial \rho_{i j}}=\left\langle\phi_{i}\left|T+V_{\mathrm{eff}}^{1}\right| \phi_{j}\right\rangle,
$$

with

$$
V_{\mathrm{eff}}^{1}=V_{H}\left[\rho_{1}\right]+V_{H}\left[\rho_{Z c}\right]+V_{x c}\left[\rho_{1} ; \rho_{c}\right]
$$

and (iii) a pseudo-on-site-contribution:

$$
\tilde{D}_{i j}^{1}=\frac{\partial \tilde{E}^{1}}{\partial \rho_{i j}}=\left\langle\tilde{\phi}_{i}\left|T+\tilde{V}_{\mathrm{eff}}^{1}\right| \tilde{\phi}_{j}\right\rangle+\sum_{L} \int_{\Omega_{\kappa}} \tilde{V}_{\mathrm{eff}}^{1}(\mathbf{r}) Q_{i j}^{L}(\mathbf{r}) d \mathbf{r},
$$

with

$$
\tilde{V}_{\text {eff }}^{1}=V_{H}\left[\tilde{\rho}_{1}+\hat{\rho}\right]+V_{H}\left[\tilde{\rho}_{Z c}\right]+V_{x c}\left[\tilde{\rho}_{1}+\hat{\rho} ; \tilde{\rho}_{c}\right],
$$

and $\Omega_{\kappa}$ is the augmentation region around atom $\kappa$.

The $\tilde{\psi}_{n}$ are not orthogonal and solve a generalized wave equation:

$$
\tilde{\mathcal{H}} \tilde{\psi}_{n}=\epsilon_{n} \mathcal{S} \tilde{\psi}_{n},
$$

where $\mathcal{S}=\mathbb{1}+\sum_{i j}\left|\tilde{p}_{i}\right\rangle s_{i j}\left\langle\tilde{p}_{j}\right|$ is the overlap operator and $\epsilon_{n}$ the eigenvalue.

\section{Density-functional perturbation theory within the projector augmented-wave approach}

Audouze et al. [11,16] have already developed a generic PAW formulation of DFPT whose notations we use hereinafter in a more synthetic way. However, this formulation does not contain any information about strains as perturbations. Because of the ultrasoft character of the PAW formalism, the pseudo-wave-functions $\tilde{\psi}_{n}$ lose their orthogonality; 
thus the Sternheimer equation [Eq. (4)] becomes

$$
\begin{aligned}
& P_{c}^{\dagger}\left(\tilde{\mathcal{H}}^{(0)}-\epsilon_{n}^{(0)} \mathcal{S}^{(0)}\right) P_{c}\left|\tilde{\psi}_{n}^{\left(\lambda_{1}\right)}\right\rangle \\
& \quad=-P_{C}^{\dagger}\left(\tilde{\mathcal{H}}^{\left(\lambda_{1}\right)}-\epsilon_{n}^{(0)} \mathcal{S}^{\left(\lambda_{1}\right)}\right)\left|\tilde{\psi}_{n}^{(0)}\right\rangle,
\end{aligned}
$$

and the associated orthogonality condition in the parallel gauge, ensured by the $P_{c}$ projectors (Eqs. (71)-(72) in Ref. [11]), is

$$
\left\langle\tilde{\psi}_{n}^{\left(\lambda_{1}\right)}\left|\mathcal{S}^{(0)}\right| \tilde{\psi}_{n}^{(0)}\right\rangle=-\frac{1}{2}\left\langle\tilde{\psi}_{n}^{(0)}\left|\mathcal{S}^{\left(\lambda_{1}\right)}\right| \tilde{\psi}_{n}^{(0)}\right\rangle .
$$

\section{Variational expression of the second-order energy changes}

The previous generalized Sternheimer equation [Eq. (24)] allows for solving a variational problem to minimize the second-order derivative of the energy, expressed as the sum of three contributions,

$$
E_{\mathrm{var}}^{\left(\lambda_{1} \lambda_{1}\right)}=E_{\tilde{\psi}^{(0)}}^{\left(\lambda_{1} \lambda_{1}\right)}+E_{\mathrm{var}, \delta \tilde{\psi}}^{\left(\lambda_{1} \lambda_{1}\right)}+E_{\mathrm{var}, H x c}^{\left(\lambda_{1} \lambda_{1}\right)}:
$$

(i) a contribution independent of the wave-function changes:

$$
E_{\tilde{\psi}^{(0)}}^{\left(\lambda_{1} \lambda_{1}\right)}=\sum_{n}\left\langle\tilde{\psi}_{n}^{(0)}\left|\frac{\partial^{2} \tilde{\mathcal{H}}}{\partial \lambda_{1}^{2}}\right|_{\tilde{\psi}^{(0)}}-\epsilon_{n} \mathcal{S}^{\left(\lambda_{1} \lambda_{1}\right)} \mid \tilde{\psi}^{(0)}\right\rangle ;
$$

(ii) a contribution explicitly dependent of the wavefunction changes,

$$
\begin{aligned}
E_{\text {var }, \delta \tilde{\psi}}^{\left(\lambda_{1} \lambda_{1}\right)}= & \sum_{n}\left\langle\tilde{\psi}_{n}^{\left(\lambda_{1}\right)}\left|\tilde{\mathcal{H}}^{(0)}-\epsilon_{n} \mathcal{S}^{(0)}\right| \tilde{\psi}_{n}^{\left(\lambda_{1}\right)}\right\rangle \\
& +\left[\left\langle\tilde{\psi}_{n}^{\left(\lambda_{1}\right)}\left|\frac{\partial \tilde{\mathcal{H}}}{\partial \lambda_{1}}\right|_{\tilde{\psi}^{(0)}}-\epsilon_{n} \mathcal{S}^{\left(\lambda_{1}\right)} \mid \tilde{\psi}_{n}^{(0)}\right\rangle+\text { c.c. }\right]
\end{aligned}
$$

and (iii) a contribution dependent of the changes in the Hartree and exchange-correlation potential, in which PAW on-site terms appear:

$$
\begin{aligned}
E_{\mathrm{var}, H x c}^{\left(\lambda_{1} \lambda_{1}\right)}= & \int_{\mathbb{R}^{3}} \int_{\mathbb{R}^{3}} \frac{1}{2} \frac{\delta^{2} E_{H x c}[\tilde{\rho} ; \hat{\rho}]}{\delta \rho(\mathbf{r}) \delta \rho\left(\mathbf{r}^{\prime}\right)} \\
& \times\left(\tilde{\rho}^{\left(\lambda_{1}\right)}(\mathbf{r})+\hat{\rho}^{\left(\lambda_{1}\right)}(\mathbf{r})\right)\left(\tilde{\rho}^{\left(\lambda_{1}\right)}\left(\mathbf{r}^{\prime}\right)+\hat{\rho}^{\left(\lambda_{1}\right)}\left(\mathbf{r}^{\prime}\right)\right) d \mathbf{r} d \mathbf{r}^{\prime} \\
& +\sum_{\kappa}\left[\int_{\Omega_{\kappa}} \int_{\Omega_{\kappa}} \frac{1}{2} \frac{\delta^{2} E_{H x c}\left[\rho_{1}\right]}{\delta \rho(\mathbf{r}) \delta \rho\left(\mathbf{r}^{\prime}\right)} \rho_{1}^{\left(\lambda_{1}\right)}(\mathbf{r}) \rho_{1}^{\left(\lambda_{1}\right)}\left(\mathbf{r}^{\prime}\right) d \mathbf{r} d \mathbf{r}^{\prime}\right. \\
& -\int_{\Omega_{\kappa}} \int_{\Omega_{\kappa}} \frac{1}{2} \frac{\delta^{2} E_{H x c}\left[\tilde{\rho}_{1} ; \hat{\rho}_{1}\right]}{\delta \rho(\mathbf{r}) \delta \rho\left(\mathbf{r}^{\prime}\right)}\left(\tilde{\rho}_{1}^{\left(\lambda_{1}\right)}(\mathbf{r})+\hat{\rho}_{1}^{\left(\lambda_{1}\right)}(\mathbf{r})\right) \\
& \left.\times\left(\tilde{\rho}_{1}^{\left(\lambda_{1}\right)}\left(\mathbf{r}^{\prime}\right)+\hat{\rho}_{1}^{\left(\lambda_{1}\right)}\left(\mathbf{r}^{\prime}\right)\right) d \mathbf{r} d \mathbf{r}^{\prime}\right] .
\end{aligned}
$$

\section{Nonvariational expression of the second-order energy changes}

We use a nonvariational form to compute the mixed second-order derivatives of the energy. This is obtained from a variational expression by canceling the changes of the wave function with respect to the second perturbation:

$$
E_{\text {nonvar }}^{\left(\lambda_{1} \lambda_{2}\right)}=E_{\tilde{\psi}^{(0)}}^{\left(\lambda_{1} \lambda_{2}\right)}+E_{\text {nonvar }, \delta \tilde{\psi}}^{\left(\lambda_{1} \lambda_{2}\right)}+E_{\text {nonvar }, H x c}^{\left(\lambda_{1} \lambda_{2}\right)} .
$$

Once again, we express this formula as a sum of three contributions: (i) a contribution independent of the wavefunction changes, identical to that in the variational formulation [Eq. (27)]; (ii) a contribution explicitly dependent of the wave-function changes:

$$
\begin{aligned}
E_{\text {nonvar }, \delta \tilde{\psi}}^{\left(\lambda_{1} \lambda_{2}\right)}= & \frac{1}{2} \sum_{n}\left[\left\langle\tilde{\psi}_{n}^{\left(\lambda_{1}\right)}\left|\tilde{\mathcal{H}}^{(0)}-\epsilon_{n} \mathcal{S}^{(0)}\right|-\frac{1}{2} \delta \tilde{\psi}_{n}^{\left(\lambda_{2}\right)}\right\rangle\right. \\
& +\left\langle\tilde{\psi}_{n}^{(0)}\left|\frac{\partial \tilde{\mathcal{H}}}{\partial \lambda_{1}}\right|_{\tilde{\psi}^{(0)}}-\epsilon_{n} \mathcal{S}^{\left(\lambda_{1}\right)} \mid-\frac{1}{2} \delta \tilde{\psi}_{n}^{\left(\lambda_{2}\right)}\right\rangle \\
& \left.+\left\langle\tilde{\psi}_{n}^{\left(\lambda_{1}\right)}\left|\frac{\partial \tilde{\mathcal{H}}}{\partial \lambda_{2}}\right|_{\tilde{\psi}^{(0)}}-\epsilon_{n} \mathcal{S}^{\left(\lambda_{2}\right)} \mid \tilde{\psi}_{n}^{(0)}\right\rangle+\text { c.c. }\right]
\end{aligned}
$$

and (iii) a contribution dependent of the changes in the Hartree and exchange-correlation potential:

$$
\begin{aligned}
& E_{\text {nonvar }, H x c}^{\left(\lambda_{1} \lambda_{2}\right)} \\
& =\int_{\mathbb{R}^{3}} \int_{\mathbb{R}^{3}} \frac{1}{2} \frac{\delta^{2} E_{H x c}[\tilde{\rho} ; \hat{\rho}]}{\delta \rho(\mathbf{r}) \delta \rho\left(\mathbf{r}^{\prime}\right)} \\
& \quad \times\left(\tilde{\rho}^{\left(\lambda_{1}\right)}(\mathbf{r})+\hat{\rho}^{\left(\lambda_{1}\right)}(\mathbf{r})\right)\left(\delta \tilde{\rho}^{\left(\lambda_{2}\right)}\left(\mathbf{r}^{\prime}\right)+\delta \hat{\rho}^{\left(\lambda_{2}\right)}\left(\mathbf{r}^{\prime}\right)\right) d \mathbf{r} d \mathbf{r}^{\prime} \\
& \quad+\sum_{\kappa}\left[\int_{\Omega_{\kappa}} \int_{\Omega_{\kappa}} \frac{1}{2} \frac{\delta^{2} E_{H x c}\left[\rho_{1}\right]}{\delta \rho(\mathbf{r}) \delta \rho\left(\mathbf{r}^{\prime}\right)} \rho_{1}^{\left(\lambda_{1}\right)}(\mathbf{r}) \delta \rho_{1}^{\left(\lambda_{2}\right)}\left(\mathbf{r}^{\prime}\right) d \mathbf{r} d \mathbf{r}^{\prime}\right. \\
& \quad-\int_{\Omega_{\kappa}} \int_{\Omega_{\kappa}} \frac{1}{2} \frac{\delta^{2} E_{H x c}\left[\tilde{\rho}_{1} ; \hat{\rho}_{1}\right]}{\delta \rho(\mathbf{r}) \delta \rho\left(\mathbf{r}^{\prime}\right)} \\
& \left.\quad \times\left(\tilde{\rho}_{1}^{\left(\lambda_{1}\right)}(\mathbf{r})+\hat{\rho}_{1}^{\left(\lambda_{1}\right)}(\mathbf{r})\right)\left(\delta \tilde{\rho}_{1}^{\left(\lambda_{2}\right)}\left(\mathbf{r}^{\prime}\right)+\delta \hat{\rho}_{1}^{\left(\lambda_{2}\right)}\left(\mathbf{r}^{\prime}\right)\right) d \mathbf{r} d \mathbf{r}^{\prime}\right] .
\end{aligned}
$$

In the above expressions, we have introduced the changes of the wave function and of the density due to the overlap operator as

$$
\delta \tilde{\psi}_{n}^{\left(\lambda_{2}\right)}=\sum_{n^{\prime}}\left\langle\tilde{\psi}_{n^{\prime}}^{(0)}\left|\mathcal{S}^{\left(\lambda_{2}\right)}\right| \tilde{\psi}_{n}^{(0)}\right| \tilde{\psi}_{n^{\prime}}^{(0)}
$$

and

$$
\delta \tilde{\rho}^{\left(\lambda_{2}\right)}(\mathbf{r})=\sum_{n}\left(\left\langle\psi_{n} \mid \mathbf{r}\right\rangle\left\langle\mathbf{r} \mid \delta \tilde{\psi}_{n}^{\left(\lambda_{2}\right)}\right\rangle+\text { c.c. }\right) .
$$

\section{The strain perturbation and the metric tensor formulation}

To treat the strain perturbation, we use the elegant metric tensor formulation proposed by Hamann et al. [3]. In both real and reciprocal spaces, all quantities are expressed using reduced coordinates and the metrics of the space. Consequently, the strain perturbation affects only the metric tensor, $\Xi_{i j}=\sum_{\alpha} R_{\alpha i}^{P} R_{\alpha j}^{P}$, where $R_{\alpha i}^{P}$ is a primitive lattice vector. The metric tensor of the reciprocal space is $\Upsilon=\Xi^{-1}$.

Then the norm of a vector $\vec{X}$ in the real space is

$$
|\vec{X}|=(\vec{X} \cdot \vec{X})^{1 / 2}=\left(\sum_{i j} \Xi_{i j} \tilde{X}_{i} \tilde{X}_{j}\right)^{1 / 2}
$$

and its derivative with respect to strain is

$$
\begin{aligned}
& \frac{\partial|\vec{X}|}{\partial \epsilon_{\alpha \beta}}=\frac{1}{2|\vec{X}|} \sum_{i j} \frac{\partial \Xi_{i j}}{\partial \epsilon_{\alpha \beta}} \tilde{X}_{i} \tilde{X}_{j}, \\
& \text { with } \frac{\partial \Xi_{i j}}{\partial \epsilon_{\alpha \beta}}=R_{\alpha i}^{P} R_{\beta j}^{P}+R_{\beta i}^{P} R_{\alpha j}^{P} .
\end{aligned}
$$


Special attention has to be paid for derivatives and integrals involving volume. Indeed, the deformation of the metrics may induce a change in volume:

$$
\frac{\partial \Omega}{\partial \epsilon_{\alpha \beta}}=\delta_{\alpha \beta} \Omega
$$

As such, an integral over the volume $\Omega$ becomes

$$
\frac{\partial}{\partial \epsilon_{\alpha \beta}}\left[\int_{\Omega} f(\mathbf{r}) d \mathbf{r}\right]=\delta_{\alpha \beta} \Omega \int_{\Omega} f(\mathbf{r}) d \mathbf{r}+\int_{\Omega} \frac{\partial f(\mathbf{r})}{\partial \epsilon_{\alpha \beta}} d \mathbf{r} .
$$

\section{E. The electric-field perturbation}

For insulators, a collective movement of atoms induces a change in the electric field. If this movement is due to an atomic vibration, the response is defined as the Born effective charge tensor [17]. If the movement is due to a strain, the response is the piezoelectric tensor [17].

To calculate these quantities within DFT, it is necessary to explicitly consider a homogeneous, static electric field in the Hamiltonian:

$$
\mathcal{H}(\mathcal{E})=\underbrace{T+V_{H x c}(\mathbf{r})+V_{\mathrm{ext}}(\mathbf{r})}_{\mathcal{H}(\mathbf{0})}+\underbrace{\mathcal{E} \cdot \hat{\mathbf{r}}}_{V_{\mathcal{E}}}
$$

where $\hat{\mathbf{r}}$ is the position operator.

Because of the electric field, the Hamiltonian loses its periodicity. The standard approach to deal with this issue is to use the long wavelength limit [18-20]. The application of the first-order Hamiltonian-at frozen wave functions- to a wave function yields the derivative of the wave function with respect to the vector $\mathbf{k}$ [15]:

$$
\left.\frac{\partial \mathcal{H}_{\mathbf{k}, \mathbf{k}}}{\partial \mathcal{E}}\right|_{\psi^{(0)}}\left|u_{n, \mathbf{k}}\right\rangle=i \frac{d\left|u_{n, \mathbf{k}}\right\rangle}{d \mathbf{k}},
$$

where $\mathcal{H}_{\mathbf{k}, \mathbf{k}}$ and $u_{n, \mathbf{k}}$ are, respectively, the Hamiltonian and the wave functions described in Bloch state space.

Thus we can reformulate the variational expression of the mixed second-order energy derivatives involving the electric field as [15]

$$
\begin{aligned}
E_{\mathrm{var}}^{\left(\lambda_{1} \mathcal{E}_{j}\right)}= & {\left[\frac { \Omega } { ( 2 \pi ) ^ { 3 } } \int d \mathbf { k } \sum _ { n } \left(\left\langle\psi_{n, \mathbf{k}}^{(0)}\left|\frac{\partial^{2} \mathcal{H}}{\partial \lambda_{1} \partial \mathcal{E}_{j}}\right|_{\psi^{(0)}} \mid \psi_{n, \mathbf{k}}^{(0)}\right\rangle\right.\right.} \\
& +\left\langle\psi_{n, \mathbf{k}}^{\left(\lambda_{1}\right)}\left|i \frac{d}{d \mathbf{k}_{j}}\right| \psi_{n, \mathbf{k}}^{(0)}\right\rangle+\left\langle\psi_{n, \mathbf{k}}^{(0)}\left|\frac{\partial \mathcal{H}}{\partial \lambda_{1}}\right|_{\psi^{(0)}} \mid \psi_{n, \mathbf{k}}^{\left(\mathcal{E}_{j}\right)}\right\rangle \\
& \left.+\left\langle\psi_{n, \mathbf{k}}^{\left(\lambda_{1}\right)}\left|\mathcal{H}^{(0)}-\epsilon_{n}^{(0)}\right| \psi_{n, \mathbf{k}}^{\left(\mathcal{E}_{j}\right)}\right\rangle\right) \\
& +\left.\frac{1}{2} \int_{\Omega} \frac{\delta V_{x c}}{\delta \rho}\right|_{\rho^{(0)}} \rho^{\left(\lambda_{1}\right)}(\mathbf{r}) \rho^{\left(\mathcal{E}_{j}\right)}(\mathbf{r}) d \mathbf{r} \\
& \left.+2 \pi \Omega \sum_{\mathbf{G} \neq 0} \frac{\rho^{\left(\lambda_{1}\right)}(\mathbf{G}) \rho^{\left(\mathcal{E}_{j}\right)}(\mathbf{G})}{G^{2}}\right]
\end{aligned}
$$

Note that we consider here the wave functions expanded in a plane-wave basis. As the derivative of the wave function with respect to $\mathbf{k}$ appears, we also need to explicitly use the Brillouin zone sampling.
From the previous equation, we can derive the two following nonvariational expressions:

$$
\begin{aligned}
E_{\text {nonvar }}^{\left(\lambda_{1} \mathcal{E}_{j}\right)}= & \frac{\Omega}{(2 \pi)^{3}} \int d \mathbf{k} \sum_{n}\left(\left\langle\psi_{n, \mathbf{k}}^{(0)}\left|\frac{\partial^{2} \mathcal{H}}{\partial \lambda_{1} \partial \mathcal{E}_{j}}\right|_{\psi^{(0)}} \mid \psi_{n, \mathbf{k}}^{(0)}\right\rangle\right. \\
& \left.+\left\langle\psi_{n, \mathbf{k}}^{\left(\lambda_{1}\right)}\left|i \frac{d}{d \mathbf{k}_{j}}\right| \psi_{n, \mathbf{k}}^{(0)}\right\rangle\right)
\end{aligned}
$$

and

$$
\begin{aligned}
E_{\text {nonvar }}^{\left(\mathcal{E}_{j} \lambda_{1}\right)}= & \frac{\Omega}{(2 \pi)^{3}} \int d \mathbf{k} \sum_{n}\left(\left\langle\psi_{n, \mathbf{k}}^{(0)}\left|\frac{\partial^{2} \mathcal{H}}{\partial \lambda_{1} \partial \mathcal{E}_{j}}\right|_{\psi^{(0)}} \mid \psi_{n, \mathbf{k}}^{(0)}\right\rangle\right. \\
& \left.+\left\langle\psi_{n, \mathbf{k}}^{(0)}\left|\frac{\partial \mathcal{H}}{\partial \lambda_{1}}\right|_{\psi^{(0)}} \mid \psi_{n, \mathbf{k}}^{\left(\mathcal{E}_{j}\right)}\right\rangle\right) .
\end{aligned}
$$

\section{F. Relaxed-ion tensors}

The perturbations with respect to field and strain are coupled to the internal degrees of freedom. As such, we can define two sets of tensors, clamped, i.e., independent on the ionic relaxations, and relaxed, i.e., dependent on the atomic relaxations. Wu et al. [17] clearly explain this coupling. For example, the action of an electric field or of strain induces a change in the atomic positions, under constraints of the symmetry. Considering the energy $E_{\mathrm{vol}}$ per unit cell volume, the elementary tensors are defined as:

the clamped-ion elastic tensor:

$$
\bar{C}_{\alpha \beta \gamma \delta}=\left.\frac{\partial^{2} E_{\mathrm{vol}}}{\partial \varepsilon_{\alpha \beta} \partial \varepsilon_{\gamma \delta}}\right|_{\tau, \mathcal{E}},
$$

the clamped-ion dielectric susceptibility tensor:

$$
\bar{\chi}_{j j^{\prime}}=-\left.\frac{\partial^{2} E_{\mathrm{vol}}}{\partial \mathcal{E}_{j} \partial \mathcal{E}_{j^{\prime}}}\right|_{\tau, \varepsilon},
$$

the clamped-ion piezoelectric tensor:

$$
\bar{e}_{j \alpha \beta}=-\left.\frac{\partial^{2} E_{\mathrm{vol}}}{\partial \mathcal{E}_{j} \partial \varepsilon_{\alpha \beta}}\right|_{\tau},
$$

the Born effective charge tensor:

$$
Z_{\kappa k j}=-\left.\Omega \frac{\partial^{2} E_{\mathrm{vol}}}{\partial \tau_{\kappa k} \partial \mathcal{E}_{j}}\right|_{\varepsilon}
$$

the clamped-ion force-strain coupling tensor:

$$
\Lambda_{\kappa k \alpha \beta}=-\left.\Omega \frac{\partial^{2} E_{\mathrm{vol}}}{\partial \tau_{\kappa k} \partial \varepsilon_{\alpha \beta}}\right|_{\mathcal{E}},
$$

and the force constant tensor:

$$
K_{\kappa k \kappa^{\prime} k^{\prime}}=\left.\Omega \frac{\partial^{2} E_{\mathrm{vol}}}{\partial \tau_{\kappa k} \partial \tau_{\kappa^{\prime} k^{\prime}}}\right|_{\mathcal{E}, \varepsilon} .
$$

Taking into account the coupling between the different perturbations and the ion relaxations, we obtain the relaxedion elastic, piezoelectric, and dielectric tensors as

$$
\begin{aligned}
C_{\alpha \beta \delta \gamma} & =\bar{C}_{\alpha \beta \delta \gamma}-\Omega^{-1} \Lambda_{\kappa k \alpha \beta}\left(K^{-1}\right)_{\kappa k \kappa^{\prime} k^{\prime}} \Lambda_{\kappa^{\prime} k^{\prime} \gamma \delta}, \\
e_{j \alpha \beta} & =\bar{e}_{j \alpha \beta}+\Omega^{-1} Z_{\kappa k j}\left(K^{-1}\right)_{\kappa k \kappa^{\prime} k^{\prime}} \Lambda_{\kappa^{\prime} k^{\prime} \alpha \beta}, \\
\chi_{j j^{\prime}} & =\bar{\chi}_{j j^{\prime}}+\Omega^{-1} Z_{\kappa k j}\left(K^{-1}\right)_{\kappa k \kappa^{\prime} k^{\prime}} Z_{\kappa^{\prime} k^{\prime} j^{\prime}} .
\end{aligned}
$$




\section{RESPONSE TO STRAIN AND ELECTRIC FIELD WITHIN THE PROJECTOR AUGMENTED-WAVE APPROACH}

In the following, we adapt the generic equations of DFPT in PAW presented in the previous section to strain and electric field perturbations. This is done in two parts. First, we calculate the second-order derivatives of the energy with respect to two strains, obtained from two contributions: one that depends on both wave functions and wave-function changes, and one that depends only on the wave functions. Second, we compute the mixed derivatives of the energy between one strain and one other perturbation.

We consider the wave functions expanded in a plane-wave basis, $\mathbf{G}$. We denote the sum of a Bloch vector $\mathbf{k}$ in the Brillouin zone and a plane-wave vector as $\mathbf{K}=\mathbf{k}+\mathbf{G} . \tilde{K}_{i}$ and $\tilde{G}_{i}$ are their corresponding components in reduced coordinates.

As a starting point, we use the formalism developed by Hamann et al. [3]. In the case of the PAW approach, it is necessary to generalize most of the formulas by Hamannet al. by introducing the following modifications: (i) the nonorthogonality of the pseudo-wave-functions, (ii) the use of a compensation charge density, (iii) the self-consistency of the nonlocal operator, and (iv) the additional on-site terms coming from contributions of the partial waves basis

Concerning the last point, contrary to the real-space grid which is used for the Fourier transform, the PAW partial wave basis is not affected by deformation. Moreover, all the on-site integrals are expressed with respect to the center of the augmentation regions, i.e., the atomic positions; as such they are not affected by displacements of the atoms resulting from deformation. Consequently, the formulas for on-site contributions to the first-order objects are not specific to the strain and are the same as for the other perturbations, like atomic displacements. All these formulas are detailed in Ref. [11]. All the derivatives of the integrals on the real-space grid contain terms coming from the metric tensor, as detailed in Sec. II D.

The second-order energy derivatives contain two parts, one involving the wave-function changes and a second one involving only ground-state wave functions. The first part is obtained from the Sternheimer equation, which needs the first-order Hamiltonian detailed in Sec. III A. The second part, at frozen wave functions, is detailed in Sec. III B.

\section{A. First-order derivatives of the Hamiltonian}

\section{Variational formulation of the second-order energy}

First we need the first-order derivatives of the pseudowave-functions $\tilde{\psi}_{n}^{(\alpha \beta)}$ with respect to the deformation $\varepsilon_{\alpha \beta}$. For this, we minimize the variational expression [Eqs. (26)(29)] of the second-order derivative of the energy using the Sternheimer equation [Eq. (24)] with $\lambda_{1}=\alpha \beta$.

The specificity of the strain perturbation lies in the derivatives of the operators, which depend now on the change of the metric tensor. For example, the kinetic operator has a nonnull derivative in the presence of strain perturbation, which is not included in Ref. [11]. The full first-order derivative of the
Hamiltonian with respect to strain becomes

$$
\begin{aligned}
\tilde{\mathcal{H}}^{(\alpha \beta)}= & T^{(\alpha \beta)}+V_{H x c}^{(\alpha \beta)}\left[\tilde{\rho} ; \hat{\rho} ; \tilde{\rho}_{c}\right]+V_{H}^{(\alpha \beta)}\left[\tilde{\rho}_{Z c}\right] \\
& +\sum_{i j}\left(\left|\tilde{p}_{i}\right\rangle \hat{D}_{i j}+D_{i j}^{1}-\tilde{D}_{i j}^{1}\left\langle\tilde{p}_{j}\right|\right)^{(\alpha \beta)} .
\end{aligned}
$$

In this framework, we explicit the various terms of the derived Hamiltonian. Some are computed in the reciprocal space and some in the real space.

\section{Contribution of the kinetic operator}

In PAW, this term is identical to the norm-conserving pseudopotential formulation, as developed by Hamann et al. [3] The derivative of the kinetic operator in a plane-wave basis only involves the derivative of the reciprocal space metric tensor $\Upsilon$ :

$$
\left\langle\tilde{\mathbf{K}}^{\prime}\left|T^{(\alpha \beta)}\right| \tilde{\mathbf{K}}\right\rangle=\delta_{\tilde{\mathbf{K}}^{\prime} \tilde{\mathbf{K}}} \frac{1}{2}\left[1+f_{\mathrm{SM}}^{\prime}\left(e_{\tilde{\mathbf{K}}}\right)\right] \sum_{i j} \Upsilon_{i j}^{(\alpha \beta)} \tilde{K}_{i} \tilde{K}_{j} .
$$

$f_{\mathrm{SM}}^{\prime}$ is the derivative of the kinetic energy smearing function $f_{\mathrm{SM}} \cdot e_{\tilde{\mathrm{K}}}$ is the kinetic energy of the plane wave.

\section{Contribution of the local potentials}

The local potential is the sum of three contributions: the local ionic pseudopotential, the Hartree potential, and the exchange and correlation potential. The three contributions corresponding to the derivative of the Hamiltonian are derived analytically in the reciprocal space. Hamann et al. [3] proposed expressions for the first-order potentials in case of strain perturbations. These expressions are directly usable in PAW by replacing the density $\rho$ with the compensated pseudodensity $\tilde{\rho}+\hat{\rho}$.

The local ionic pseudopotential $V_{H}\left[\tilde{\rho}_{Z c}\right](\mathbf{r})$ is the sum of the radial contributions $v_{H}^{\kappa}\left[\tilde{\rho}_{Z c}\right]\left(\left|\mathbf{r}-\boldsymbol{\tau}_{\kappa}\right|\right)$ of the atoms located in $\boldsymbol{\tau}_{\kappa}$. Its corresponding derivative in the reciprocal space with respect to strain is [3]

$$
\begin{aligned}
V_{H}\left[\tilde{\rho}_{Z c}\right]^{(\alpha \beta)}(\mathbf{G})= & \sum_{\kappa} e^{-2 i \pi \mathbf{G} \cdot \tau_{\kappa}}\left[-\delta_{\alpha \beta} v_{H}^{\kappa}\left[\tilde{\rho}_{Z c}\right](G)\right. \\
& \left.+\frac{v_{H}^{\kappa}\left[\tilde{\rho}_{Z c}\right]^{\prime}(G)}{2 G} \sum_{i j} \Upsilon_{i j}^{(\alpha \beta)} \tilde{G}_{i} \tilde{G}_{j}\right] .
\end{aligned}
$$

$v_{H}^{\kappa}\left[\tilde{\rho}_{Z c}\right](G)$ is the radial Fourier transform of the atomic potential and $v_{H}^{\kappa}\left[\tilde{\rho}_{Z c}\right]^{\prime}(G)$ is its derivative.

The Hartree potential, explicitly involving the compensation charge density specific to PAW, is derived with respect to a deformation as [3]

$$
\begin{aligned}
V_{H}[\tilde{\rho}+\hat{\rho}]^{(\alpha \beta)}(\mathbf{G}) \\
=\frac{4 \pi}{G^{2}}\left[\left(\tilde{\rho}^{(\alpha \beta)}(\mathbf{G})+\hat{\rho}^{(\alpha \beta)}(\mathbf{G})\right)\right. \\
\left.\quad-(\tilde{\rho}(\mathbf{G})+\hat{\rho}(\mathbf{G}))\left(\delta_{\alpha \beta}+\frac{1}{G^{2}} \sum_{i j} \Upsilon_{i j}^{(\alpha \beta)} \tilde{G}_{i} \tilde{G}_{j}\right)\right] .
\end{aligned}
$$


The derivatives of the densities are detailed below (Sec. III A 5).

The exchange and correlation potential contains a nonlinear core correction which involves the pseudo-core-density $\tilde{\rho}_{c}(\mathbf{r})$ [21]. Its derivative with respect to strain contains a volume contribution, as well as contributions from core, valence, and compensation charge densities. In the LDA framework, it is

$$
\begin{aligned}
V_{x c}^{(\alpha \beta)}(\mathbf{r})= & K_{x c}(\mathbf{r})\left[-\delta_{\alpha \beta}(\tilde{\rho}(\mathbf{r})+\hat{\rho}(\mathbf{r}))\right. \\
& \left.+\frac{\partial \tilde{\rho}_{\mathrm{c}}(\mathbf{r})}{\partial \varepsilon_{\alpha \beta}}+\left(\tilde{\rho}^{(\alpha \beta)}(\mathbf{r})+\hat{\rho}^{(\alpha \beta)}(\mathbf{r})\right)\right],
\end{aligned}
$$

with

$$
K_{x c}(\mathbf{r})=\left.\frac{\delta V_{x c}[\rho]}{\delta \rho(\mathbf{r})}\right|_{\rho^{(0)}} .
$$

The pseudo-core-density $\tilde{\rho}_{c}(\mathbf{r})$ is the sum of the radial atomic contributions $\tilde{\rho}_{c}^{\kappa}\left(\left|\mathbf{r}-\boldsymbol{\tau}_{\kappa}\right|\right)$, expressed in the reciprocal space (unlike Ref. [3]), as

$$
\tilde{\rho}_{c}(\mathbf{G})=\sum_{\kappa} \tilde{\rho}_{c}^{\kappa}(G) e^{-2 i \pi \mathbf{G} \cdot \tau_{\kappa}}
$$

Its corresponding derivative in the reciprocal space with respect to strain is obtained as in the case of the local ionic pseudopotential as

$$
\begin{aligned}
\frac{\partial \tilde{\rho}_{\mathrm{c}}(\mathbf{G})}{\partial \varepsilon_{\alpha \beta}}= & \frac{1}{\Omega} \sum_{\kappa} e^{-2 i \pi \mathbf{G} \cdot \tau_{\kappa}}\left[-\delta_{\alpha \beta} \tilde{\rho}_{c}^{\kappa}(G)\right. \\
& \left.+\frac{\tilde{\rho}_{c}^{\kappa},(G)}{2 G} \sum_{i j} \Upsilon_{i j}^{(\alpha \beta)} \tilde{G}_{i} \tilde{G}_{j}\right] .
\end{aligned}
$$

$\tilde{\rho}_{c}^{\kappa}(G)$ is the radial Fourier transform of the pseudo-coredensity and $\tilde{\rho}_{c}^{\kappa},(G)$ is its derivative. The limit of $\frac{\tilde{\rho}_{c}^{\kappa}(G)}{G}$ in $G=0$ is detailed in the Appendix.

\section{Contribution of the nonlocal potential}

In the PAW formalism, the nonlocal potential, $V_{N L}$ is self-consistently determined from the pseudo-wave-functions. Thus, its derivative with respect to strain contains two parts, one from the projectors and one from the intensities $D_{i j}$ :

$$
V_{N L}^{(\alpha \beta)} 7=\sum_{i j}\left[\left(\left|\tilde{p}_{i}\right\rangle\left\langle\tilde{p}_{j}\right|\right)^{(\alpha \beta)} D_{i j}+\left|\tilde{p}_{i}\right\rangle\left(D_{i j}\right)^{(\alpha \beta)}\left\langle\tilde{p}_{j}\right|\right] .
$$

The derivatives with respect to strain of the projectors $\left(\left|\tilde{p}_{i}\right\rangle\left\langle\tilde{p}_{j}\right|\right)^{(\alpha \beta)}$ are detailed in the Appendix. These derivatives are in principle analogous to those obtained in normconserving pseudopotentials by Hamman et al. [3]. But the formalism based on the Legendre polynomials cannot be generalized in PAW because of the off-diagonal terms of $D_{i j}$. This is why we need to express this potential in terms of spherical harmonics.

The $D_{i j}$ derivatives are made of three contributions:

(i) A contribution from the compensation charge density:

$$
\begin{aligned}
\left(\hat{D}_{i j}\right)^{(\alpha \beta)}= & \sum_{L} \int_{\mathbb{R}^{3}}\left[\tilde{V}_{\mathrm{eff}}^{(\alpha \beta)}(\mathbf{r}) Q_{i j}^{L}(\mathbf{r})\right. \\
& \left.+\tilde{V}_{\mathrm{eff}}(\mathbf{r})\left(\left(Q_{i j}^{L}\right)^{(\alpha \beta)}(\mathbf{r})+\delta_{\alpha \beta} Q_{i j}^{L}(\mathbf{r})\right)\right] d \mathbf{r} .
\end{aligned}
$$

The last term arises from the metric change [Eq. (38)]. The different parts of $\tilde{V}_{\text {eff }}^{(\alpha \beta)}$ (local, Hartree, and exchangecorrelation) have been detailed in the previous section. The integral over $\mathbb{R}^{3}$ implies derivatives of the metric tensor.

(ii) An all-electron on-site contribution:

$$
\left(D_{i j}^{1}\right)^{(\alpha \beta)}=\left\langle\phi_{i}\left|\left(V_{\mathrm{eff}}^{1}\right)^{(\alpha \beta)}\right| \phi_{j}\right\rangle .
$$

(iii) A pseudo-on-site-contribution:

$$
\begin{aligned}
\left(\tilde{D}_{i j}^{1}\right)^{(\alpha \beta)}= & \left\langle\tilde{\phi}_{i}\left|\left(\tilde{V}_{\mathrm{eff}}^{1}\right)^{(\alpha \beta)}\right| \tilde{\phi}_{j}\right\rangle+\sum_{L} \int_{\Omega_{\kappa}}\left[\left(\tilde{V}_{\mathrm{eff}}^{1}\right)^{(\alpha \beta)}(\mathbf{r}) Q_{i j}^{L}(\mathbf{r})\right. \\
& \left.+\tilde{V}_{\mathrm{eff}}^{1}(\mathbf{r})\left(Q_{i j}^{L}\right)^{(\alpha \beta)}(\mathbf{r})\right] d \mathbf{r}
\end{aligned}
$$

As we stated at the beginning of this section, these expressions do not require any special treatment for strain perturbation. As such, they are detailed in Eqs. (78)-(79) of Ref. [11].

Note that the treatment of the overlap operator $S$ is very similar to the nonlocal potential one and contains only the derivatives of the projectors:

$$
S^{(\alpha \beta)}=\sum_{i j} s_{i j}\left(\left|\tilde{p}_{i}\right\rangle\left\langle\tilde{p}_{j}\right|\right)^{(\alpha \beta)} \text {. }
$$

\section{Derivatives of the densities}

The derivatives of the different PAW density components with respect to strain are straightforwardly expressed as

$$
\begin{aligned}
& \tilde{\rho}^{(\alpha \beta)}(\mathbf{r})=\sum_{n} f_{n}\left(\tilde{\psi}_{n}^{*(\alpha \beta)}(\mathbf{r}) \tilde{\psi}_{n}^{(0)}(\mathbf{r})+\tilde{\psi}_{n}^{*(0)}(\mathbf{r}) \tilde{\psi}_{n}^{(\alpha \beta)}(\mathbf{r})\right) \\
& \rho_{1}^{(\alpha \beta)}(\mathbf{r})=\sum_{i j} \rho_{i j}^{(\alpha \beta)} \phi_{i}(\mathbf{r}) \phi_{j}(\mathbf{r}) \\
& \tilde{\rho}_{1}^{(\alpha \beta)}(\mathbf{r})=\sum_{i j} \rho_{i j}^{(\alpha \beta)} \tilde{\phi}_{i}(\mathbf{r}) \tilde{\phi}_{j}(\mathbf{r}) \\
& \hat{\rho}^{(\alpha \beta)}(\mathbf{r})=\sum_{i j L}\left[\rho_{i j}^{(\alpha \beta)} Q_{i j}^{L}(\mathbf{r})+\rho_{i j}\left(Q_{i j}^{L}\right)^{(\alpha \beta)}(\mathbf{r})\right]
\end{aligned}
$$

with the derivative of the occupation matrix given by

$$
\begin{aligned}
\rho_{i j}^{(\alpha \beta)}= & \sum_{n} f_{n}\left\langle\tilde{\psi}_{n}^{(\alpha \beta)} \mid \tilde{p}_{i}\right\rangle\left\langle\tilde{p}_{j} \mid \tilde{\psi}_{n}^{(0)}\right\rangle+\left\langle\tilde{\psi}_{n}^{(0)} \mid \tilde{p}_{i}\right\rangle\left\langle\tilde{p}_{j} \mid \tilde{\psi}_{n}^{(\alpha \beta)}\right\rangle \\
& +\left\langle\tilde{\psi}_{n}^{(0)}\left|\left(\left|\tilde{p}_{i}\right\rangle\left\langle\tilde{p}_{j}\right|\right)^{(\alpha \beta)}\right| \tilde{\psi}_{n}^{(0)}\right\rangle .
\end{aligned}
$$

All these formulas do not reveal specificities related to the response to strain, except for the moments $Q_{i j}^{L}(\mathbf{r})$ of the density of charge compensation:

$$
\left(Q_{i j}^{l m}\right)^{(\alpha \beta)}(\mathbf{r})=q_{i j}^{l} \frac{d\left(g_{l}\left(\left|\mathbf{r}-\boldsymbol{\tau}_{\kappa}\right|\right) Y_{l m}\left(\widehat{\mathbf{r}-\boldsymbol{\tau}_{\kappa}}\right)\right)}{d\left(\mathbf{r}-\boldsymbol{\tau}_{\kappa}\right)} \cdot \frac{d\left(\mathbf{r}-\boldsymbol{\tau}_{\kappa}\right)}{d \varepsilon_{\alpha \beta}} .
$$

Combining the strain tensor symmetry and the metric tensor formulation this derivative can be rewritten as

$$
\begin{aligned}
\left(Q_{i j}^{l m}\right)^{(\alpha \beta)}(\mathbf{r})= & \frac{1}{2} q_{i j}^{l}\left[\left(\mathbf{r}-\boldsymbol{\tau}_{\kappa}\right)_{\beta} \frac{d\left(g_{l}\left(\left|\mathbf{r}-\boldsymbol{\tau}_{\kappa}\right|\right) Y_{l m}\left(\widehat{\mathbf{r}-\boldsymbol{\tau}_{\kappa}}\right)\right)}{d\left(\mathbf{r}-\boldsymbol{\tau}_{\kappa}\right)_{\alpha}}\right. \\
& \left.+\left(\mathbf{r}-\boldsymbol{\tau}_{\kappa}\right)_{\alpha} \frac{d\left(g_{l}\left(\left|\mathbf{r}-\boldsymbol{\tau}_{\kappa}\right|\right) Y_{l m}\left(\widehat{\mathbf{r}-\boldsymbol{\tau}_{\kappa}}\right)\right)}{d\left(\mathbf{r}-\boldsymbol{\tau}_{\kappa}\right)_{\beta}}\right] .
\end{aligned}
$$




\section{B. Second-order derivatives of the energy not involving the wave-function changes}

We discuss next the different terms of the second-order energy derivatives at frozen wave functions $\tilde{\psi}_{n}^{(0)}$, which are found in both the variational and the nonvariational expressions:

$$
E_{\tilde{\psi}_{n}^{(0)}}^{(\alpha \beta \gamma \delta)}=\left.\frac{1}{2} \frac{\partial^{2} E}{\partial \varepsilon_{\alpha \beta} \partial \varepsilon_{\gamma \delta}}\right|_{\tilde{\psi}^{(0)}}=\frac{1}{2} \sum_{n}\left\langle\tilde{\psi}_{n}^{(0)}\left|\frac{\partial^{2} \tilde{\mathcal{H}}}{\partial \varepsilon_{\alpha \beta} \partial \varepsilon_{\gamma \delta}}\right|_{\tilde{\psi}^{(0)}}-\epsilon_{n} \mathcal{S}^{(\alpha \beta \gamma \delta)} \mid \tilde{\psi}_{n}^{(0)}\right\rangle .
$$

\section{Contribution of the kinetic operator}

Similar to Sec. III A 2, this PAW term is identical to the norm-conserving pseudopotential formulation, as developed by Hamann et al. [3]. The second-order derivative of the kinetic energy only involves the derivatives of the reciprocal space metric tensor $\Upsilon$ :

$$
\left.\frac{\partial^{2} E_{T}}{\partial \varepsilon_{\alpha \beta} \partial \varepsilon_{\gamma \delta}}\right|_{\tilde{\psi}^{(0)}}=\sum_{n}\left\langle\tilde{\psi}_{n}^{(0)}\left|T^{(\alpha \beta \gamma \delta)}\right| \tilde{\psi}_{n}^{(0)}\right\rangle,
$$

with the derivative of the kinetic operator expressed in the reciprocal space as

$$
\left\langle\tilde{\mathbf{K}}^{\prime}\left|T^{(\alpha \beta \gamma \delta)}\right| \tilde{\mathbf{K}}_{\rangle}=\delta_{\tilde{\mathbf{K}}^{\prime} \tilde{\mathbf{K}}}\left(f_{\text {SM }}^{\prime \prime}\left(e_{\tilde{\mathbf{K}}}\right)\left[\frac{1}{2} \sum_{i j} \Upsilon_{i j}^{(\alpha \beta)} \tilde{K}_{i} \tilde{K}_{j}\right]^{2}+\frac{1}{2}\left[1+f_{\text {SM }}^{\prime}\left(e_{\tilde{\mathbf{K}}}\right)\right]^{2} \sum_{i j} \Upsilon_{i j}^{(\alpha \beta \gamma \delta)} \tilde{K}_{i} \tilde{K}_{j}\right) .\right.
$$

$f_{\mathrm{SM}}^{\prime \prime}$ is the second derivative of the kinetic energy smearing function $f_{\mathrm{SM}}$.

\section{Contribution of the local potentials}

Once more, the expressions for the strain derivatives of the local $E_{\mathrm{loc}}$, Hartree $E_{H}$, and exchange-correlation $E_{x c}$ energies were developed by Hamann et al. [3]. For PAW, we replace the density $\rho$ with the compensated pseudodensity $\tilde{\rho}+\hat{\rho}$ :

$$
\begin{gathered}
\left.\frac{\partial^{2} E_{l o c}}{\partial \varepsilon_{\alpha \beta} \partial \varepsilon_{\gamma \delta}}\right|_{\tilde{\psi}^{(0)}}=\sum_{\mathbf{G} \neq 0}(\tilde{\rho}(\mathbf{G})+\hat{\rho}(\mathbf{G})) \sum_{\kappa} e^{-2 i \pi \mathbf{G} \cdot \tau_{\kappa}}\left[\delta_{\alpha \beta} \delta_{\gamma \delta} v_{H}^{\kappa}\left[\tilde{\rho}_{Z c}\right](G)+\sum_{i j} \Upsilon_{i j}^{(\alpha \beta)} \tilde{G}_{i} \tilde{G}_{j}\right. \\
\left.\sum_{k l} \Upsilon_{k l}^{(\gamma \delta)} \tilde{G}_{k} \tilde{G}_{l}\left(\frac{v_{H}^{\kappa}\left[\tilde{\rho}_{Z c}\right]^{\prime \prime}(G)}{4 G^{2}}-\frac{v_{H}^{\kappa}\left[\tilde{\rho}_{Z c}\right]^{\prime}(G)}{4 G^{3}}\right)-\frac{v_{H}^{\kappa}\left[\tilde{\rho}_{Z c}\right]^{\prime}(G)}{2 G} \sum_{i j}\left(\delta_{\alpha \beta} \Upsilon_{i j}^{(\gamma \delta)}+\delta_{\gamma \delta} \Upsilon_{i j}^{(\alpha \beta)}-\Upsilon_{i j}^{(\alpha \beta \gamma \delta)}\right) \tilde{G}_{i} \tilde{G}_{j}\right] \\
\left.\frac{\partial^{2} E_{H}}{\partial \varepsilon_{\alpha \beta} \partial \varepsilon_{\gamma \delta}}\right|_{\tilde{\psi}^{(0)}}=2 \pi \Omega \sum_{\mathbf{G} \neq 0}|(\tilde{\rho}(\mathbf{G})+\hat{\rho}(\mathbf{G}))|^{2}\left[G^{-2} \delta_{\alpha \beta} \delta_{\gamma \delta}+G^{-4} \sum_{i j}\left(\delta_{\alpha \beta} \Upsilon_{i j}^{(\gamma \delta)}+\delta_{\gamma \delta} \Upsilon_{i j}^{(\alpha \beta)}-\Upsilon_{i j}^{(\alpha \beta \gamma \delta)} \tilde{G}_{i} \tilde{G}_{j}\right)\right. \\
\left.+2 G^{-6} \sum_{i j} \Upsilon_{i j}^{(\gamma \delta)} \tilde{G}_{i} \tilde{G}_{j} \sum_{k l} \Upsilon_{k l}^{(\gamma \delta)} \tilde{G}_{k} \tilde{G}_{l}\right], \\
\left.\frac{\partial^{2} E_{x c}}{\partial \varepsilon_{\alpha \beta} \partial \varepsilon_{\gamma \delta}}\right|_{\tilde{\psi}^{(0)}}=\delta_{\alpha \beta} \delta_{\gamma \delta} E_{x c}+\Omega \int\left[V_{x c}(\mathbf{r}) \frac{\partial^{2} \tilde{\rho}_{c}(\mathbf{r})}{\partial \varepsilon_{\alpha \beta} \partial \varepsilon_{\gamma \delta}}+\left(K_{x c}(\mathbf{r})(\tilde{\rho}(\mathbf{r})+\hat{\rho}(\mathbf{r}))-V_{x c}(\mathbf{r})\right)\right. \\
\times\left(\delta_{\alpha \beta} \delta_{\gamma \delta}(\tilde{\rho}(\mathbf{r})+\hat{\rho}(\mathbf{r}))-\delta_{\alpha \beta} \frac{\partial \tilde{\rho}_{c}(\mathbf{r})}{\partial \varepsilon_{\gamma \delta}}-\delta_{\gamma \delta} \frac{\partial \tilde{\rho}_{c}(\mathbf{r})}{\partial \varepsilon_{\alpha \beta}}\right)+K_{x c}(\mathbf{r}) \frac{\partial \tilde{\rho}_{c}(\mathbf{r})}{\partial \varepsilon_{\gamma \delta}} \frac{\partial \tilde{\rho}_{c}(\mathbf{r})}{\left.\partial \varepsilon_{\alpha \beta}\right] d \mathbf{r} .}
\end{gathered}
$$

The second-order derivative of the pseudo-core-density $\tilde{\rho}_{c}$ is straightforwardly obtained from the first-order one [Eq. (58)]. As in the case of the first-order derivative, these equations are developed in the reciprocal space, unlike Ref. [3]:

$$
\begin{aligned}
\frac{\partial^{2} \tilde{\rho}_{c}(\mathbf{G})}{\partial \varepsilon_{\alpha \beta} \partial \varepsilon_{\gamma \delta}}= & \frac{1}{\Omega} \sum_{a} e^{-2 i \pi \mathbf{G} \cdot \tau_{\kappa}}\left[\delta_{\alpha \beta} \delta_{\gamma \delta} \tilde{\rho}_{c}(G)-\delta_{\alpha \beta} \frac{\tilde{\rho}_{c}^{\kappa \prime}(G)}{2 G} \sum_{i j} \Upsilon_{i j}^{(\gamma \delta)} \tilde{G}_{i} \tilde{G}_{j}-\delta_{\gamma \delta} \frac{\tilde{\rho}_{c}^{\kappa}(G)}{2 G} \sum_{i j} \Upsilon_{i j}^{(\alpha \beta)} \tilde{G}_{i} \tilde{G}_{j}\right. \\
& \left.+\left(\frac{\tilde{\rho}_{c}^{\kappa \prime \prime}(G)}{4 G^{2}}-\frac{\tilde{\rho}_{c}^{\kappa^{\prime}}(G)}{4 G^{3}}\right) \sum_{i j} \Upsilon_{i j}^{(\alpha \beta)} \tilde{G}_{i} \tilde{G}_{j} \sum_{k l} \Upsilon_{k l}^{(\gamma \delta)} \tilde{G}_{k} \tilde{G}_{l}+\frac{\tilde{\rho}_{c}^{\kappa \prime}(G)}{2 G} \sum_{i j} \Upsilon_{i j}^{(\alpha \beta \gamma \delta)} \tilde{G}_{i} \tilde{G}_{j},\right]
\end{aligned}
$$

where $\tilde{\rho}_{c}^{\kappa}$ '” $(G)$ is the second-order derivative of the pseudo-core-density in reciprocal space. The limits of $\frac{\tilde{\rho}_{c}^{\kappa}(G)}{G}$ and $\left(\frac{\tilde{\rho}_{c}^{\kappa \prime \prime}(G)}{G^{2}}-\right.$ $\left.\frac{\tilde{\rho}_{c}^{\kappa^{\prime}}(G)}{G^{3}}\right)$ at $G=0$ are given in the Appendix.

The extension of this formalism to the generalized gradient approximation requires the transformation of the equations related to the pseudo-core-density presented in Ref. [17] to the reciprocal space. 


\section{Contribution of the nonocal potential}

The second-order derivative of the nonlocal operator contains the most novelties because of the self-consistency of the $D_{i j}$ scalars. It is solved similarly to the first-order one. At frozen wave function, it contains only derivatives of the projectors and of the $D_{i j}$ scalars. Only the integral over the real-space grid, i.e., the $\hat{D}_{i j}$ term, contributes to the derivatives of the scalars. At each additional order of the derivation, new terms appear from the metrics changes. Only the derivatives of the local pseudopotential $V_{H}\left[\tilde{\rho}_{Z c}\right]\left[\right.$ Eq. (53)] intervene in the derivation of the effective potential $\tilde{V}_{\text {eff }}$.

The first-order derivative of the nonlocal energy becomes

$$
\begin{aligned}
\left.\frac{\partial E_{\mathrm{nl}}}{\partial \varepsilon_{\alpha \beta}}\right|_{\tilde{\psi}^{(0)}}= & \int_{\mathbb{R}^{3}}\left(V_{H}\left[\tilde{\rho}_{Z c}\right]\right)^{(\alpha \beta)}(\mathbf{r}) \hat{\rho}(\mathbf{r}) d \mathbf{r}+\sum_{i j} \sum_{n} f_{n}\left\langle\tilde{\psi}_{n}\left|\frac{\partial\left|\tilde{p}_{i}\right\rangle\left\langle\tilde{p}_{j}\right|}{\partial \varepsilon_{\alpha \beta}}\right| \tilde{\psi}_{n}\right\rangle\left(D_{i j}-\varepsilon_{n} s_{i j}\right) \\
& +\sum_{i j l m} \rho_{i j} \int_{\mathbb{R}^{3}}\left[\tilde{V}_{\mathrm{eff}}(\mathbf{r})\left(Q_{i j}^{l m}\right)^{(\alpha \beta)}(\mathbf{r})+\delta_{\alpha \beta} \tilde{V}_{\mathrm{eff}}(\mathbf{r}) Q_{i j}^{l m}(\mathbf{r})\right] d \mathbf{r} .
\end{aligned}
$$

The next derivative is

$$
\begin{aligned}
& \frac{\partial^{2} E_{\mathrm{nl}}}{\left.\partial \varepsilon_{\gamma \delta} \partial \varepsilon_{\alpha \beta}\right|_{\tilde{\psi}^{(0)}}=} \int_{\mathbb{R}^{3}}\left(V_{H}\left[\tilde{\rho}_{Z c}\right]\right)^{(\alpha \beta \gamma \delta)}(\mathbf{r}) \hat{\rho}(\mathbf{r}) d \mathbf{r}+\sum_{i j} \sum_{n} f_{n}\left\langle\tilde{\psi}_{n}\left|\frac{\partial^{2}\left|\tilde{p}_{i}\right\rangle\left\langle\tilde{p}_{j}\right|}{\partial \varepsilon_{\alpha \beta} \partial \varepsilon_{\gamma \delta}}\right| \tilde{\psi}_{n}\right\rangle\left(D_{i j}-\varepsilon_{n} s_{i j}\right) \\
&+\sum_{i j l m} \rho_{i j} \int_{\mathbb{R}^{3}}\left[\tilde{V}_{\mathrm{eff}}(\mathbf{r})\left(Q_{i j}^{l m}\right)^{(\alpha \beta \gamma \delta)}(\mathbf{r})+\left(V_{H}\left[\tilde{\rho}_{Z c}\right]\right)^{(\alpha \beta)}(\mathbf{r})\left(Q_{i j}^{l m}\right)^{(\gamma \delta)}(\mathbf{r})\right] d \mathbf{r} \\
&+\sum_{i j l m} \rho_{i j} \int_{\mathbb{R}^{3}}\left[\left(V_{H}\left[\tilde{\rho}_{Z c}\right]\right)^{(\gamma \delta)}(\mathbf{r})\left(Q_{i j}^{l m}\right)^{(\alpha \beta)}(\mathbf{r})+\delta_{\alpha \beta}\left(V_{H}\left[\tilde{\rho}_{Z c}\right]\right)^{(\gamma \delta)}(\mathbf{r}) Q_{i j}^{l m}(\mathbf{r})\right] d \mathbf{r} \\
&+\sum_{i j l m} \rho_{i j} \int_{\mathbb{R}^{3}}\left[\delta_{\gamma \delta}\left(V_{H}\left[\tilde{\rho}_{Z c}\right]\right)^{(\alpha \beta)}(\mathbf{r}) Q_{i j}^{l m}(\mathbf{r})+\delta_{\alpha \beta} \tilde{V}_{\mathrm{eff}}(\mathbf{r})\left(Q_{i j}^{l m}\right)^{(\alpha \beta)}(\mathbf{r})\right] d \mathbf{r} \\
&+\sum_{i j l m} \rho_{i j} \int_{\mathbb{R}^{3}}\left[\delta_{\gamma \delta} \tilde{V}_{\mathrm{eff}}(\mathbf{r})\left(Q_{i j}^{l m}\right)^{(\alpha \beta)}(\mathbf{r})+\delta_{\gamma \delta} \delta_{\alpha \beta} \tilde{V}_{\mathrm{eff}}(\mathbf{r}) Q_{i j}^{l m}(\mathbf{r})\right] d \mathbf{r} \\
&+\left.\sum_{i j l m} \frac{\partial \rho_{i j}}{\partial \varepsilon_{\gamma \delta}}\right|_{\tilde{\psi}^{(0)}} \int_{\mathbb{R}^{3}}\left[\tilde{V}_{\mathrm{eff}}(\mathbf{r})\left(Q_{i j}^{l m}\right)^{(\alpha \beta)}(\mathbf{r})+\left(V_{H}\left[\tilde{\rho}_{Z c}\right]\right)^{(\alpha \beta)}(\mathbf{r}) Q_{i j}^{l m}(\mathbf{r})+\delta_{\alpha \beta} \tilde{V}_{\mathrm{eff}}(\mathbf{r}) Q_{i j}^{l m}(\mathbf{r})\right] d \mathbf{r} \\
&+\left.\sum_{i j l m} \frac{\partial \rho_{i j}}{\partial \varepsilon_{\alpha \beta}}\right|_{\tilde{\psi}^{(0)}} \int_{\mathbb{R}^{3}}\left[\tilde{V}_{\mathrm{eff}}(\mathbf{r})\left(Q_{i j}^{l m}\right)^{(\alpha \beta)}(\mathbf{r})+\left(V_{H}\left[\tilde{\rho}_{Z c}\right]\right)^{(\gamma \delta)}(\mathbf{r}) Q_{i j}^{l m}(\mathbf{r})+\delta_{\gamma \delta} \tilde{V}_{\mathrm{eff}}(\mathbf{r}) Q_{i j}^{l m}(\mathbf{r})\right] d \mathbf{r},
\end{aligned}
$$

with $\left.\frac{\partial \rho_{i j}}{\partial \varepsilon_{\alpha \beta}}\right|_{\tilde{\psi}^{(0)}}=\sum_{n} f_{n}\left\langle\tilde{\psi}_{n}\left|\frac{\partial\left|\tilde{p}_{i}\right\rangle\left\langle\tilde{p}_{j}\right|}{\partial \varepsilon_{\alpha \beta}}\right| \tilde{\psi}_{n}\right\rangle$, The second-order derivatives of the projectors are given in the Appendix. The secondorder derivatives of the moments $Q_{i j}^{l m}(\mathbf{r})$ are obtained straightforwardly as in Sec. III A 5:

$$
\begin{aligned}
\left(Q_{i j}^{l m}\right)^{(\alpha \beta \gamma \delta)}(\mathbf{r})= & \frac{1}{4} q_{i j}^{l}\left[\delta_{\gamma \beta}\left(\mathbf{r}-\boldsymbol{\tau}_{\kappa}\right)_{\delta} \frac{d\left(g_{l}\left(\left|\mathbf{r}-\boldsymbol{\tau}_{\kappa}\right|\right) Y_{l m}\left(\widehat{\mathbf{r}-\boldsymbol{\tau}_{\kappa}}\right)\right)}{d\left(\mathbf{r}-\boldsymbol{\tau}_{\kappa}\right)_{\alpha}}+\left(\mathbf{r}-\boldsymbol{\tau}_{\kappa}\right)_{\beta}\left(\mathbf{r}-\boldsymbol{\tau}_{\kappa}\right)_{\gamma} \frac{d^{2}\left(g_{l}\left(\left|\mathbf{r}-\boldsymbol{\tau}_{\kappa}\right|\right) Y_{l m}\left(\widehat{\mathbf{r}-\boldsymbol{\tau}_{\kappa}}\right)\right)}{d\left(\mathbf{r}-\boldsymbol{\tau}_{\kappa}\right)_{\delta} d\left(\mathbf{r}-\boldsymbol{\tau}_{\kappa}\right)_{\alpha}}\right. \\
& +\delta_{\gamma \alpha}\left(\mathbf{r}-\boldsymbol{\tau}_{\kappa}\right)_{\delta} \frac{d\left(g_{l}\left(\left|\mathbf{r}-\boldsymbol{\tau}_{\kappa}\right|\right) Y_{l m}\left(\widehat{\mathbf{r}-\boldsymbol{\tau}_{\kappa}}\right)\right)}{d\left(\mathbf{r}-\boldsymbol{\tau}_{\kappa}\right)_{\beta}}+\left(\mathbf{r}-\boldsymbol{\tau}_{\kappa}\right)_{\alpha}\left(\mathbf{r}-\boldsymbol{\tau}_{\kappa}\right)_{\gamma} \frac{d^{2}\left(g_{l}\left(\left|\mathbf{r}-\boldsymbol{\tau}_{\kappa}\right|\right) Y_{l m}\left(\widehat{\mathbf{r}-\boldsymbol{\tau}_{\kappa}}\right)\right)}{d\left(\mathbf{r}-\boldsymbol{\tau}_{\kappa}\right)_{\delta} d\left(\mathbf{r}-\boldsymbol{\tau}_{\kappa}\right)_{\beta}} \\
& +\delta_{\delta \beta}\left(\mathbf{r}-\boldsymbol{\tau}_{\kappa}\right)_{\gamma} \frac{d\left(g_{l}\left(\left|\mathbf{r}-\boldsymbol{\tau}_{\kappa}\right|\right) Y_{l m}\left(\widehat{\mathbf{r}-\boldsymbol{\tau}_{\kappa}}\right)\right)}{d\left(\mathbf{r}-\boldsymbol{\tau}_{\kappa}\right)_{\alpha}}+\left(\mathbf{r}-\boldsymbol{\tau}_{\kappa}\right)_{\beta}\left(\mathbf{r}-\boldsymbol{\tau}_{\kappa}\right)_{\delta} \frac{d^{2}\left(g_{l}\left(\left|\mathbf{r}-\boldsymbol{\tau}_{\kappa}\right|\right) Y_{l m}\left(\widehat{\mathbf{r}-\boldsymbol{\tau}_{\kappa}}\right)\right)}{d\left(\mathbf{r}-\boldsymbol{\tau}_{\kappa}\right)_{\gamma} d\left(\mathbf{r}-\boldsymbol{\tau}_{\kappa}\right)_{\alpha}} \\
& \left.+\delta_{\delta \alpha}\left(\mathbf{r}-\boldsymbol{\tau}_{\kappa}\right)_{\gamma} \frac{d\left(g_{l}\left(\left|\mathbf{r}-\boldsymbol{\tau}_{\kappa}\right|\right) Y_{l m}\left(\widehat{\mathbf{r}-\boldsymbol{\tau}_{\kappa}}\right)\right)}{d\left(\mathbf{r}-\boldsymbol{\tau}_{\kappa}\right)_{\beta}}+\left(\mathbf{r}-\boldsymbol{\tau}_{\kappa}\right)_{\alpha}\left(\mathbf{r}-\boldsymbol{\tau}_{\kappa}\right)_{\delta} \frac{d^{2}\left(g_{l}\left(\left|\mathbf{r}-\boldsymbol{\tau}_{\kappa}\right|\right) Y_{l m}\left(\widehat{\mathbf{r}-\boldsymbol{\tau}_{\kappa}}\right)\right)}{d\left(\mathbf{r}-\boldsymbol{\tau}_{\kappa}\right)_{\gamma} d\left(\mathbf{r}-\boldsymbol{\tau}_{\kappa}\right)_{\beta}}\right]
\end{aligned}
$$

\section{Nonvariational form of the second-order derivative of the energy}

Once the derivative of the wave function with respect to strain is computed, we can use the nonvariational expression of the second-order energy [Eq. (5)] to obtain the different mixed derivatives. In our case, this allows us to obtain the clamped-ion elastic tensor $\bar{C}_{\alpha \beta \gamma \delta}$, the clamped-ion force-strain coupling tensor $\Lambda_{\kappa k \alpha \beta}$, and the clamped-ion piezoelectric tensor $\bar{e}_{j \alpha \beta}$.

\section{The clamped-ion elastic tensor}

The clamped-ion elastic tensor corresponds to the second derivative of the energy with respect to only two strains. To obtain it, we need to consider $\bar{C}_{\alpha \beta \gamma \delta}=\frac{2}{\Omega} E_{\text {nonvar }}^{(\alpha \beta \gamma \delta)}$ and 
use Eqs. (30)-(32). Here, everything was already detailed in the previous sections, except the first-order derivative of the Hamiltonian with respect to strain at frozen wave functions. This latter part is

$$
\begin{aligned}
\left.\frac{\partial \tilde{\mathcal{H}}}{\partial \varepsilon_{\alpha \beta}}\right|_{\tilde{\psi}^{(0)}}= & T^{(\alpha \beta)}+\left(V_{H}\left[\tilde{\rho}_{Z c}\right]\right)^{(\alpha \beta)}+\sum_{i j}\left(\left|\tilde{p}_{i}\right\rangle\left\langle\tilde{p}_{j}\right|\right)^{(\alpha \beta)} D_{i j} \\
& +\sum_{i j}\left(\left|\tilde{p}_{i}\right\rangle\left\langle\tilde{p}_{j}\right|\right) \sum_{l m} \int_{\mathbb{R}^{3}}\left[\left(V_{H}\left[\tilde{\rho}_{Z c}\right]\right)^{(\alpha \beta)}(\mathbf{r}) Q_{i j}^{l m}(\mathbf{r})\right. \\
& \left.+\tilde{V}_{\mathrm{eff}}(\mathbf{r})\left(Q_{i j}^{l m}(\mathbf{r})\right)^{(\alpha \beta)}\right] d \mathbf{r} .
\end{aligned}
$$

\section{The clamped-ion force-strain coupling tensor}

The clamped-ion force-strain coupling tensor corresponds to the second derivative of the energy with respect to one strain and one atomic displacement. To obtain it, we need to consider $\Lambda_{\kappa k \alpha \beta}=-2 E_{\text {nonvar }}^{\left(\alpha \beta \tau_{\kappa k}\right)}$ and use again Eqs. (30)-(32). Here the sign comes from Eq. (48) and the factor 2 from the Taylor expansion. At this point, the only unknown terms are the first-order derivatives of the Hamiltonian with respect to one atomic displacement and the second-order derivative of the energy with respect to one strain and one atomic displacement, both at frozen wave functions.

The first-order Hamiltonian at frozen wave functions is

$$
\begin{aligned}
\left.\frac{\partial \tilde{\mathcal{H}}}{\partial \tau_{\kappa k}}\right|_{\tilde{\psi}^{(0)}}= & \left(V_{H}\left[\tilde{\rho}_{Z c}\right]\right)^{\left(\tau_{\kappa k}\right)}+\sum_{i j}\left(\left|\tilde{p}_{i}\right\rangle\left\langle\tilde{p}_{j}\right|\right)^{\left(\tau_{\kappa k}\right)} D_{i j} \\
& +\sum_{i j}\left(\left|\tilde{p}_{i}\right\rangle\left\langle\tilde{p}_{j}\right|\right) \sum_{l m} \int_{\mathbb{R}^{3}}\left[\left(V_{H}\left[\tilde{\rho}_{Z c}\right]\right)^{\left(\tau_{\kappa k}\right)}(\mathbf{r}) Q_{i j}^{l m}(\mathbf{r})\right. \\
& \left.+\tilde{V}_{\mathrm{eff}}(\mathbf{r})\left(Q_{i j}^{l m}(\mathbf{r})\right)^{\left(\tau_{\kappa k}\right)}\right] d \mathbf{r}
\end{aligned}
$$

In this expression, the terms derived with respect to atomic displacements have the same form as in the DFPT formalism without strain and have already been detailed in the literature $[11,18,22,23]$.

The second-order energy at frozen wave functions $E_{\tilde{\psi}_{n}^{(0)}}^{\left(\alpha \beta \tau_{k k}\right)}$ contains the local pseudopotential, exchange-correlation, and nonlocal potential contributions. The former two are similar to those proposed by Ref. [3] by replacing the density $\rho$ with the compensated pseudodensity $\tilde{\rho}+\hat{\rho}$ :

$$
\begin{gathered}
\left.\frac{\partial^{2} E_{\mathrm{loc}}}{\partial \varepsilon_{\alpha \beta} \partial \tau_{\kappa k}}\right|_{\tilde{\psi}^{(0)}}=-2 \pi i \sum_{\mathbf{G} \neq 0}(\tilde{\rho}(\mathbf{G})+\hat{\rho}(\mathbf{G})) \mathbf{G} e^{-2 i \pi \mathbf{G} \cdot \tau_{\kappa}}\left[-\delta_{\alpha \beta} v_{H}^{k}\left[\rho_{Z c}\right](G)+\frac{v_{H}^{k}\left[\rho_{Z c}\right]^{\prime}(G)}{2 G} \sum_{i j} \Upsilon_{i j}^{(\alpha \beta)} \tilde{G}_{i} \tilde{G}_{j}\right] \\
\left.\frac{\partial^{2} E_{x c}}{\partial \varepsilon_{\alpha \beta} \partial \tau_{\kappa k}}\right|_{\tilde{\psi}^{(0)}}=\Omega \int\left[V_{x c}(\mathbf{r}) \frac{\partial^{2} \tilde{\rho}_{c}(\mathbf{r})}{\partial \varepsilon_{\alpha \beta} \tau_{\kappa k}}+K_{x c}(\mathbf{r}) \frac{\partial \tilde{\rho}_{c}(\mathbf{r})}{\partial \varepsilon_{\alpha \beta}} \frac{\partial \tilde{\rho}_{c}(\mathbf{r})}{\partial \tau_{\kappa k}}+\delta_{\alpha \beta}\left(V_{x c}(\mathbf{r})-K_{x c}(\mathbf{r})(\tilde{\rho}(\mathbf{r})+\hat{\rho}(\mathbf{r})) \frac{\partial \tilde{\rho}_{c}(\mathbf{r})}{\partial \tau_{\kappa k}}\right] d \mathbf{r},\right.
\end{gathered}
$$

where the derivatives of the pseudo-core-density are expressed in the reciprocal space as

$$
\frac{\partial \tilde{\rho}_{c}(\mathbf{G})}{\partial \tau_{\kappa k}}=-2 i \pi \mathbf{G} \rho_{c}^{\kappa},(G) e^{-2 i \pi \mathbf{G} \cdot \tau_{\kappa}} \Omega^{-1}
$$

and

$$
\frac{\partial^{2} \tilde{\rho}_{c}^{\kappa}(\mathbf{G})}{\varepsilon_{\alpha \beta} \tau_{\kappa k}}=-2 i \mathbf{G} \pi\left[-\delta_{\alpha \beta} \tilde{\rho}_{c}^{\kappa}(G)+\frac{\tilde{\rho}_{c}^{\kappa},(G)}{2 G} \sum_{i j} \Upsilon_{i j}^{(\alpha \beta)} \tilde{G}_{i} \tilde{G}_{j}\right] e^{-2 i \pi \mathbf{G} \cdot \tau_{\kappa}} \Omega^{-1} .
$$

Similar to the second-order derivative with respect to two strains, the contribution due to the nonlocal potential can be written as

$$
\begin{aligned}
\left.\frac{\partial^{2} E_{\mathrm{nl}}}{\partial \varepsilon_{\alpha \beta} \partial \tau_{\kappa k}}\right|_{\tilde{\psi}^{(0)}}= & \int_{\mathbb{R}^{3}}\left(V_{H}\left[\tilde{\rho}_{Z c}\right]\right)^{\left(\alpha \beta \tau_{\kappa k}\right)}(\mathbf{r}) \hat{\rho}(\mathbf{r}) d \mathbf{r}+\sum_{i j} \sum_{n} f_{n}\left\langle\tilde{\psi}_{n}\left|\frac{\partial^{2}\left|\tilde{p}_{i}\right\rangle\left\langle\tilde{p}_{j}\right|}{\partial \varepsilon_{\alpha \beta} \partial \tau_{\kappa k}}\right| \tilde{\psi}_{n}\right\rangle\left(D_{i j}-\varepsilon_{n} s_{i j}\right) \\
& +\sum_{i j l m} \rho_{i j} \int_{\mathbb{R}^{3}}\left[\left(V_{H}\left[\tilde{\rho}_{Z c}\right]\right)^{\left(\tau_{k k}\right)}(\mathbf{r})\left(Q_{i j}^{l m}\right)^{(\alpha \beta)}(\mathbf{r})+\tilde{V}_{\mathrm{eff}}(\mathbf{r})\left(Q_{i j}^{l m}\right)^{\left(\alpha \beta \tau_{k k}\right)}(\mathbf{r})+\left.\frac{\partial \tilde{V}_{\mathrm{eff}}(\mathbf{r})}{\partial \varepsilon_{\alpha \beta}}\right|_{\tilde{\psi}^{(0)}}\left(Q_{i j}^{l m}\right)^{\left(\tau_{k k}\right)}(\mathbf{r})\right] d \mathbf{r} \\
& +\sum_{i j l m} \rho_{i j} \int_{\mathbb{R}^{3}}\left[\delta_{\alpha \beta}\left(V_{H}\left[\tilde{\rho}_{Z c}\right]\right)^{\left(\tau_{k k}\right)}(\mathbf{r}) Q_{i j}^{l m}(\mathbf{r})+\delta_{\alpha \beta} \tilde{V}_{\mathrm{eff}}(\mathbf{r})\left(Q_{i j}^{l m}\right)^{\left(\tau_{k k}\right)}(\mathbf{r})\right] d \mathbf{r} \\
& +\left.\sum_{i j l m} \frac{\partial \rho_{i j}}{\partial \tau_{\kappa k}}\right|_{\tilde{\psi}^{(0)}} \int_{\mathbb{R}^{3}}\left[\tilde{V}_{\mathrm{eff}}\left(Q_{i j}^{l m}\right)^{(\alpha \beta)}(\mathbf{r})+\delta_{\alpha \beta} \tilde{V}_{\mathrm{eff}}(\mathbf{r}) Q_{i j}^{l m}(\mathbf{r})+\left.\frac{\partial \tilde{V}_{\mathrm{eff}}(\mathbf{r})}{\partial \varepsilon_{\alpha \beta}}\right|_{\tilde{\psi}^{(0)}} Q_{i j}^{l m}(\mathbf{r})\right] d \mathbf{r} \\
& +\left.\sum_{i j l m} \frac{\partial \rho_{i j}}{\partial \varepsilon_{\alpha \beta}}\right|_{\tilde{\psi}^{(0)}} \int_{\mathbb{R}^{3}}\left[\left(V_{H}\left[\tilde{\rho}_{Z c}\right]\right)^{\left(\tau_{k k}\right)}(\mathbf{r}) Q_{i j}^{l m}(\mathbf{r})+\tilde{V}_{\mathrm{eff}}(\mathbf{r})\left(Q_{i j}^{l m}\right)^{\left(\tau_{k k}\right)}(\mathbf{r})\right] d \mathbf{r},
\end{aligned}
$$

with $\left.\frac{\partial \rho_{i j}}{\partial \tau_{k k}}\right|_{\tilde{\psi}^{(0)}}=\sum_{n} f_{n}\left\langle\tilde{\psi}_{n}\left|\frac{\partial\left|\tilde{p}_{i}\right\rangle\left\langle\tilde{p}_{j}\right|}{\partial \tau_{k k}}\right| \tilde{\psi}_{n}\right\rangle$.

The second-order derivatives of the projectors are given in the Appendix. 
The second-order derivatives of the moments $Q_{i j}^{l m}(\mathbf{r})$ are

$$
\begin{aligned}
\left(Q_{i j}^{l m}\right)^{\left(\alpha \beta \tau_{\kappa k}\right)}(\mathbf{r})= & \frac{1}{2} q_{i j}^{l}\left[-\delta_{k \beta} \frac{d\left(g_{l}\left(\left|\mathbf{r}-\boldsymbol{\tau}_{\kappa}\right|\right) Y_{l m}\left(\widehat{\mathbf{r}-\boldsymbol{\tau}_{\kappa}}\right)\right)}{d\left(\mathbf{r}-\boldsymbol{\tau}_{\kappa}\right)_{\alpha}}-\delta_{k \alpha} \frac{d\left(g_{l}\left(\left|\mathbf{r}-\boldsymbol{\tau}_{\kappa}\right|\right) Y_{l m}\left(\widehat{\mathbf{r}-\boldsymbol{\tau}_{\kappa}}\right)\right)}{d\left(\mathbf{r}-\boldsymbol{\tau}_{\kappa}\right)_{\beta}}\right. \\
& \left.+\left(\mathbf{r}-\boldsymbol{\tau}_{\kappa}\right)_{\beta} \frac{d^{2}\left(g_{l}\left(\left|\mathbf{r}-\boldsymbol{\tau}_{\kappa}\right|\right) Y_{l m}\left(\widehat{\mathbf{r}-\boldsymbol{\tau}_{\kappa}}\right)\right)}{d\left(\mathbf{r}-\boldsymbol{\tau}_{\kappa}\right)_{k} d\left(\mathbf{r}-\boldsymbol{\tau}_{\kappa}\right)_{\alpha}}+\left(\mathbf{r}-\boldsymbol{\tau}_{\kappa}\right)_{\alpha} \frac{d^{2}\left(g_{l}\left(\left|\mathbf{r}-\boldsymbol{\tau}_{\kappa}\right|\right) Y_{l m}\left(\widehat{\mathbf{r}-\boldsymbol{\tau}_{\kappa}}\right)\right)}{d\left(\mathbf{r}-\boldsymbol{\tau}_{\kappa}\right)_{k} d\left(\mathbf{r}-\boldsymbol{\tau}_{\kappa}\right)_{\beta}}\right] .
\end{aligned}
$$

\section{The clamped-ion tensors involving the electric field}

The perturbation with respect to electric field is different than the other perturbations. Starting from Eqs. (30)-(32), we can obtain two different nonvariational expressions for the mixed derivatives between one homogeneous electric field and one other perturbation, and compute the dielectric tensor, the Born effective charges, and the piezoelectric tensor.

In case of derivatives with respect to electric field, there are no derivatives of the overlap operator; consequently, there is no corresponding $\delta \widetilde{\psi}_{n}^{\left(\mathcal{E}_{j}\right)}$ and $\delta \tilde{\rho}^{\left(\mathcal{E}_{j}\right)}$ contributions.

First, we start with $\lambda_{1}=\mathcal{E}_{j}$ and $\lambda_{2}=\tau_{\kappa k}, \alpha \beta$ or $\mathcal{E}_{j^{\prime}}$. This yields a nonvariational expression similar to Eqs. (30)-(32), which requires the derivative of the pseudo-wave-functions with respect to the electric field $\tilde{\psi}_{n}^{\left(\mathcal{(}_{j}\right)}$.

Then we consider $\lambda_{1}=\tau_{\kappa k}, \alpha \beta$ or $\mathcal{E}_{j^{\prime}}$ and $\lambda_{2}=\mathcal{E}_{j}$. This yields a different expression, which is simpler and requires the derivative of the pseudo-wave-functions with respect to $\lambda_{1}, \tilde{\psi}_{n}^{\left(\lambda_{1}\right)}$ :

$$
\begin{aligned}
E_{\text {nonvar }}^{\left(\lambda_{1} \mathcal{E}_{j}\right)}= & \sum_{n}\left[\left\langle\tilde{\psi}_{n}^{(0)}\left|\frac{\partial^{2} \tilde{\mathcal{H}}}{\partial \lambda_{1} \partial \mathcal{E}_{j}}\right|_{\tilde{\psi}^{(0)}} \mid \tilde{\psi}_{n}^{(0)}\right\rangle\right. \\
& \left.+\left\langle\tilde{\psi}_{n}^{\left(\lambda_{1}\right)}\left|\frac{\partial \tilde{\mathcal{H}}}{\partial \mathcal{E}_{j}}\right|_{\tilde{\psi}^{(0)}} \mid \tilde{\psi}_{n}^{(0)}\right\rangle+\text { c.c. }\right] .
\end{aligned}
$$

At this point, the only unknown terms are the first-order derivatives of the Hamiltonian with respect to the electric field and the second-order derivative of the energy with respect to one electric field and one other perturbation, both at frozen wave functions.

To compute the first-order Hamiltonian, we combine the method of the long wavelength limit and the application of the PAW transformation. This yields a formula for the first-order Hamiltonian in the Bloch state space that is identical to the formulation in ultrasoft pseudopotentials $[19,20]$, because the $k$-point grid does not cover the PAW on-site terms. Using PAW notations, this formula is rewritten as

$$
\begin{aligned}
\left.\frac{\partial \tilde{\mathcal{H}}_{\mathbf{k}, \mathbf{k}}}{\partial \mathcal{E}}\right|_{\tilde{\psi}^{(0)}}\left|u_{n, \mathbf{k}}\right\rangle & =\underbrace{\left[\mathbb{1}+\sum_{i, i^{\prime}}\left|\tilde{p}_{i, \mathbf{k}}\right\rangle s_{i i^{\prime}}\left\langle\tilde{p}_{i^{\prime}, \mathbf{k}}\right|\right]}_{\mathcal{S}_{\mathbf{k}, \mathbf{k}}} i \frac{d}{d \mathbf{k}}\left|u_{n, \mathbf{k}}\right\rangle \\
& +\quad \sum_{i, i^{\prime}}\left|\tilde{p}_{i, \mathbf{k}}\right\rangle \chi_{i i^{\prime}}\left\langle\tilde{p}_{i^{\prime}, \mathbf{k}} \mid u_{n, \mathbf{k}}\right\rangle \\
& +\quad \sum_{i, i^{\prime}}\left|\tilde{p}_{i, \mathbf{k}}\right\rangle s_{i i^{\prime}}\left\langle i \frac{d \tilde{p}_{i^{\prime}, \mathbf{k}}}{d \mathbf{k}} \mid u_{n, \mathbf{k}}\right\rangle,
\end{aligned}
$$

with

$$
\begin{aligned}
& \chi_{i i^{\prime}}=-\left(\left\langle\phi_{i}\left|\mathbf{r}-\boldsymbol{\tau}_{\kappa}\right| \phi_{i^{\prime}}\right\rangle-\left\langle\tilde{\phi}_{i}\left|\mathbf{r}-\boldsymbol{\tau}_{\kappa}\right| \tilde{\phi}_{i^{\prime}}\right\rangle\right), \\
& s_{i i^{\prime}}=\left\langle\phi_{i} \mid \phi_{i^{\prime}}\right\rangle-\left\langle\tilde{\phi}_{i} \mid \tilde{\phi}_{i^{\prime}}\right\rangle .
\end{aligned}
$$

The expressions of the operators and vectors in the Bloch states space are

$$
\begin{aligned}
\tilde{\mathcal{H}}_{\mathbf{k}, \mathbf{k}^{\prime}} & =e^{-i \mathbf{k} \cdot \mathbf{r}} \tilde{\mathcal{H}} e^{i \mathbf{k}^{\prime} \cdot \mathbf{r}^{\prime}}, \\
\mathcal{S}_{\mathbf{k}, \mathbf{k}^{\prime}} & =e^{-i \mathbf{k} \cdot \mathbf{r}} \mathcal{S} e^{i \mathbf{k}^{\prime} \cdot \mathbf{r}^{\prime}}, \\
\left|\phi_{i, \mathbf{k}}\right\rangle & =e^{-i \mathbf{k} \cdot\left(\mathbf{r}-\boldsymbol{\tau}_{\kappa}\right)}\left|\phi_{i}\right\rangle, \\
\left|\tilde{\phi}_{i, \mathbf{k}}\right\rangle & =e^{-i \mathbf{k} \cdot\left(\mathbf{r}-\boldsymbol{\tau}_{\kappa}\right)}\left|\tilde{\phi}_{i}\right\rangle \\
\left|\tilde{p}_{i, \mathbf{k}}\right\rangle & =e^{-i \mathbf{k} \cdot\left(\mathbf{r}-\boldsymbol{\tau}_{\kappa}\right)}\left|\tilde{p}_{i}\right\rangle .
\end{aligned}
$$

To compute the second-order derivative of the energy at frozen wave functions, we simply derivate $\sum_{n}\left\langle\tilde{\psi}_{n}^{(0)}\left|\frac{\partial \tilde{\mathcal{H}}}{\partial \mathcal{E}}\right|_{\tilde{\psi}^{(0)}} \mid \tilde{\psi}_{n}^{(0)}\right\rangle$ with respect to another perturbation $\lambda_{1}$ :

$$
\begin{aligned}
\sum_{n}\left\langle\tilde{\psi}_{n}^{(0)}\left|\frac{\partial^{2} \tilde{\mathcal{H}}}{\partial \mathcal{E}_{j} \partial \lambda_{1}}\right|_{\tilde{\psi}^{(0)}} \mid \tilde{\psi}_{n}^{(0)}\right\rangle \\
=2 \frac{\Omega}{(2 \pi)^{3}} \int_{B Z} \sum_{n}\left[\left\langle\tilde{\psi}_{n}^{(0)}\right| \sum_{i i^{\prime}} s_{i i^{\prime}} \frac{\partial}{\partial \lambda_{1}}\left(\left|\tilde{p}_{i}\right\rangle i \frac{\partial\left\langle\tilde{p}_{i^{\prime}}\right|}{\partial k_{j}}\right)\left|\tilde{\psi}_{n}^{(0)}\right\rangle\right. \\
\quad+\left\langle\tilde{\psi}_{n}^{(0)}\left|\sum_{i i^{\prime}}\left(\chi_{i i^{\prime}}\right)_{j} \frac{\partial\left|\tilde{p}_{i}\right\rangle\left\langle\tilde{p}_{i^{\prime}}\right|}{\partial \lambda_{1}}\right| \tilde{\psi}_{n}^{(0)}\right\rangle \\
\left.\quad+\left\langle\tilde{\psi}_{n}^{(0)}\left|\sum_{i i^{\prime}} s_{i i^{\prime}} \frac{\partial\left|\tilde{p}_{i}\right\rangle\left\langle\tilde{p}_{i^{\prime}}\right|}{\partial \lambda_{1}}\right| i \frac{\partial \tilde{\psi}_{n}^{(0)}}{\partial k_{j}}\right\rangle\right] d \mathbf{k} .
\end{aligned}
$$

The derivatives of the projectors $\tilde{p}_{i}$ are given in the Appendix.

\section{VALIDATION}

\section{A. Validation procedure}

In the ABINIT software package [24-26], the DFPT formalism was already available within the PAW approach, and the elastic formalism within the norm-conserving pseudopotential approach [17]. Here we have extended the DFPT implementation within PAW to include the elastic response, according to the formulas of the previous sections. In the following, we discuss numerical aspects of the implementation, and compare results obtained with the two implementations of the nonstationary expressions [Eqs. (42) and (43)]. Then we compare the DFPT approach with the finite difference (FD) one on several high-symmetry systems. We end with applications on two realistic low-symmetry systems.

We perform the FD calculations of the strain and electric field derivatives using a five-point formula and sufficiently small deformations to ensure a constant set of K-points. We thoroughly converge our calculations in terms of cutoff 
energies and Brillouin zone sampling. We especially check for the charge neutrality as an efficient indication of convergence. When available we provide also experimental values.

\section{B. Numerical issues}

The practical implementation aspects of the computation of the energy derivatives are highly dependent of the formalism. Consequently direct numerical comparisons of the results are highly challenging. This is particularly true when the derivatives concern deformations combined with atomic relaxations and electric fields. The multiple interdependencies of the various terms, as shown in the big Hessian matrix [27], may easily induce numerical errors.

In FD we obtain the elastic tensor assuming linear stressstrain relations. We apply positive and negative deformations with $\epsilon$ and $2 \epsilon$ amplitude. The value of $\epsilon$ has to be carefully chosen as it directly controls the precision of the test. It has to be sufficiently small to ensure that we are in the linear regime but not too small to avoid round-off errors. In case of a significant discrepancy between the DFPT and the FD approaches, the amplitude of the deformation in FD is the first parameter that has to be questioned. Its optimal value is strongly system dependent and relies on several factors like the amplitude of the atomic relaxations.

The deformation of the system due to the application of a strain generates a change of the real-space grid used to define the integrals centered on the atoms and to represent the scalar fields, like densities and potentials. This grid is usually defined in reduced coordinates related to the primitive vector of the unit cell. Its deformation can induce large fluctuations for the integrals. Actually, this lays at the origin of the Pulay contribution to the stresses, a side effect of the grid spacing changes under strain. In the present case, it makes the numerical check of the integrals and of the partial derivatives of scalar fields very complicated. The latter have to be maintained constant which is not easy with an evolving grid. An interpolation allows to match the deformed grid to the original one.

In order to validate the implementation of the effective charges and of the piezoelectric tensors by FD it is necessary to take a different approach because of the electric field specificities. There are two alternatives: (i) compute the polarizability on different perturbed cells, or (ii) compute small variations of the forces and/or stresses on cells under varying electric field. In both cases, it is necessary to carry out PAW ground state calculations in the formalism of the "Modern Theory of Polarization", as implemented in ABINIT [9]. Such calculations are known to have a poor convergence behavior with respect to the k-point sampling because they include a numerical computation of the derivative of the wavefunction with respect to the k-points. Therefore it is difficult to get a perfect agreement between DFPT and FD, as shown on Fig 1.

\section{Numerical validation on highly symmetric systems}

Tables I, II, and III show, respectively, a partial set of the internal-strain coupling parameters, the Born effective charges and the clamped-ion piezoelectric tensor of AlAs. They have been computed using the two nonvariational expressions of the second-order energy and converged up to between 6 and 9 significant digits. As shown by these tables these two ex-

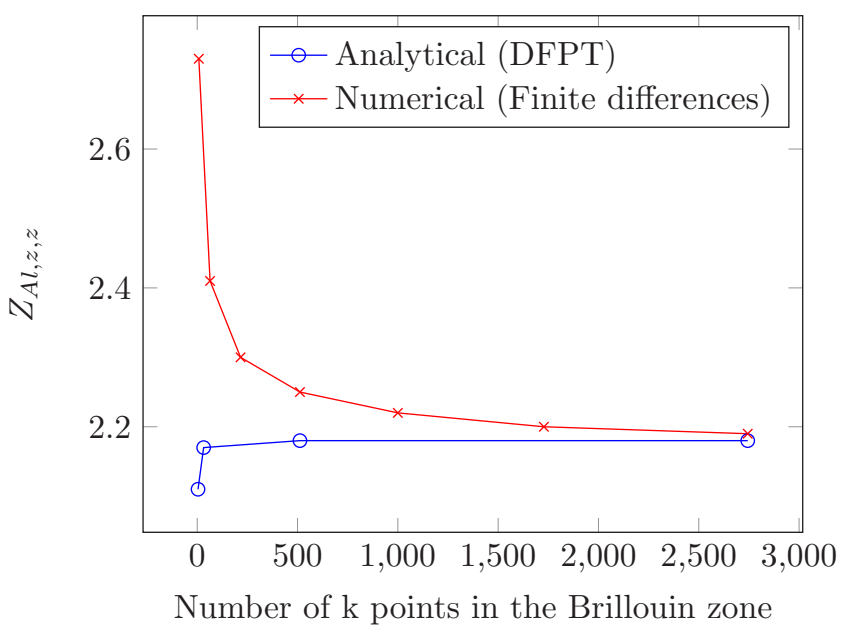

FIG. 1. Convergence of the effective charges in AlAs with respect to the Brillouin zone sampling as obtained in both finite differences and in DFPT.

pressions give the same values, up to many enough significant digits to validate the formulas and their implementation.

Table IV lists the three independent values of the elastic tensor of FCC aluminum, obtained with DFPT and FD, clearly showing the agreement between the two approaches.

In this test involving a metal, we use a specific treatment of the Fermi energy [28], not discussed in this paper. Indeed, changes of the lattice parameters affect the grid of G-points and thus the occupancy of the electronic bands around the Fermi energy.

\section{Validation on low-symmetry systems}

To make the validation more complete we consider a structure with lower symmetry: C-bearing $\epsilon$-iron, a structure that can potentially be present in the Earth's solid inner core. [30] We build a $2 \times 2 \times 1$ super-cell of the hexagonal close packed (hcp) structure of $\epsilon$-iron, containing $16 \mathrm{Fe}$ atoms and we place one interstitial carbon atom. The symmetry is reduced from hexagonal $\left(\mathrm{P}_{3} m m c\right)$ to trigonal $(\mathrm{P}-3 m 1)$, and all the atoms need to be relaxed as they do not occupy anymore special positions fixed by the symmetry.

The determination of the full elastic tensor requires computation of 7 independent elastic constants. In FD appropriate

TABLE I. Clamped-ion force-strain coupling parameters of AlAs in reduced coordinates, in Ha. Comparison of the numerical values obtained with DFPT and FD: $-\Omega \frac{\partial^{2} E_{\mathrm{vol}}}{\partial \tau_{\kappa k} \partial \varepsilon_{\alpha \beta}}$ [Eq. (48)].

\begin{tabular}{lllrr}
\hline \hline$\varepsilon_{\alpha \beta}$ & $\kappa$ & $k$ & \multicolumn{1}{c}{ FD+PAW } & \multicolumn{1}{c}{ DFPT + PAW } \\
\hline 1 & $\mathrm{Al}$ & $\mathrm{x}$ & 0.84452759 & 0.84452677 \\
2 & $\mathrm{Al}$ & $\mathrm{x}$ & -0.84452759 & -0.84452679 \\
3 & $\mathrm{Al}$ & $\mathrm{x}$ & 0.00000000 & 0.00000000 \\
4 & $\mathrm{Al}$ & $\mathrm{x}$ & -0.40239908 & -0.40239911 \\
5 & $\mathrm{Al}$ & $\mathrm{x}$ & -0.69697561 & -0.69697569 \\
6 & $\mathrm{Al}$ & $\mathrm{x}$ & -0.48758797 & -0.48758777 \\
\hline \hline
\end{tabular}


TABLE II. Born effective charges of AlAs in units of charge [Eq. (47)]. Comparison between the two nonvariational expressions, Eqs. (30)-(32) and Eq. (86), in DFPT.

\begin{tabular}{llrr}
\hline \hline$\kappa$ & $k$ & $-\Omega \frac{\partial^{2} E_{\mathrm{vol}}}{\partial \tau_{\kappa k} \partial \overrightarrow{\mathcal{E}}_{k}}$ & \multicolumn{1}{c}{$-\Omega \frac{\partial^{2} E_{\mathrm{vol}}}{\partial \overrightarrow{\mathcal{E}}_{k} \partial \tau_{\kappa k}}$} \\
\hline $\mathrm{Al}$ & $\mathrm{x}$ & 2.07482486 & 2.07482525 \\
$\mathrm{Al}$ & $\mathrm{y}$ & 2.07482486 & 2.07482525 \\
$\mathrm{Al}$ & $\mathrm{Z}$ & 2.22353351 & 2.22353327 \\
As & $\mathrm{x}$ & -2.07485811 & -2.07485849 \\
As & $\mathrm{y}$ & -2.07485811 & -2.07485849 \\
As & $\mathrm{Z}$ & -2.22341484 & -2.22341461 \\
\hline \hline
\end{tabular}

strain systems need to be applied where all the atoms need to be relaxed.

The relaxed-ion elastic tensors obtained from DFPT and FD are shown in Table V. The differences between the values obtained with the two approaches are on the order of tens of MPa or less, two orders of magnitude smaller than the typical error bars in this type of simulations.

Then we consider the $\mathrm{MgSiO}_{3}$ perovskite, the mineral bridgmanite. It is by volume the most important mineral of the Earth. It forms more than three quarters of the Earth's lower mantle - a spherical shell inside our planet that extends between about 660-km depth and 2900-km depth. Since its discovery various groups of both computational and experimental researchers have extensively studied its physical properties, with a special regard on the elasticity [27]. As all natural minerals, this is not a pure phase, with most notably $\mathrm{Fe}^{2+}, \mathrm{Fe}^{3+}$, and $\mathrm{Al}^{3+}$ as major substitutions on the $\mathrm{Mg}$ and/or Si sites.

Here we determine the elastic tensors of pure $\mathrm{MgSiO}_{3}$ bridgmanite at pressures corresponding to the Earth's lower mantle. This is just to show how efficient the DFPT+PAW method is for the determination of elastic properties on realistic materials with direct applications.

$\mathrm{MgSiO}_{3}$ bridgmanite has a distorted perovskite structure with Pnma space group and $Z=4$ formula units per unit cell. We employ a $8 \times 8 \times 8$ grid of special $\mathbf{k}$ points, a kinetic energy cutoff of $30 \mathrm{Ha}$ for the plane waves on the real-space grid, and of $60 \mathrm{Ha}$ for the grid inside the augmentation regions.

Table VI compares the elastic constants tensor computed using various methods with the experimental values. The values obtained in PAW, in both DFPT and FD, are strikingly similar. They are also globally closer to the experimental values than the ones obtained within norm-conserving.

TABLE III. Piezoelectric tensor of AlAs in units of charge/Bohr ${ }^{2}$ [Eq. (46)]. Comparison between the two nonvariational expressions, Eqs. (30)-(32) and Eq. (86), in DFPT.

\begin{tabular}{llrr}
\hline \hline$\varepsilon_{\alpha \beta}$ & $k$ & $-\frac{\partial^{2} E_{\mathrm{vol}}}{\partial \varepsilon_{\alpha \beta} \overrightarrow{\mathcal{E}}_{k}}$ & $-\frac{\partial^{2} E_{\mathrm{vol}}}{\partial \overrightarrow{\mathcal{E}}_{k} \partial \varepsilon_{\alpha \beta}}$ \\
\hline 5 & $\mathrm{X}$ & 0.01036757 & 0.01036757 \\
4 & $\mathrm{y}$ & 0.01036986 & 0.01036831 \\
1 & $\mathrm{Z}$ & 0.00645215 & 0.00645173 \\
2 & $\mathrm{Z}$ & 0.00645215 & 0.00645173 \\
3 & $\mathrm{Z}$ & -0.00991493 & -0.00991383 \\
\hline \hline
\end{tabular}

TABLE IV. Elastic tensor of fcc Al in GPa, obtained with DFPT and FD.

\begin{tabular}{lccl}
\hline \hline Elastic constant & $C_{11}$ & $C_{12}$ & $C_{44}$ \\
\hline FD & 114.36042 & 60.01343 & 34.00284 \\
DFPT & 114.35981 & 60.01364 & 34.00364 \\
Exp. [29] & 114.30 & 61.92 & 31.62 \\
\hline \hline
\end{tabular}

\section{E. Numerical efficiency of the DFPT implementation}

Next we estimate and compare the total walltime necessary to obtain the full elastic tensor in FD and DFPT. For this, we consider the $\mathrm{Fe}_{16} \mathrm{C}$ unit cell, at $320 \mathrm{GPa}$ with 17 atoms per unit cell and trigonal symmetry. As a result, we need to compute seven independent elastic constants. All simulations were performed on 512 CPUs, on the Curie supercomputer at the French TGCC supercomputing center.

For the FD simulations, this comes down to performing a minimum of 29 independent structural relaxations, using a simple 5-points derivative formula. Each of these relaxations requires about $1400 \mathrm{CPU}$ walltime hours. This makes a total walltime of about 40000 CPU hours.

For the DFPT simulations, this comes down to performing one structural relaxation (same $1400 \mathrm{CPU}$ hours), followed by one full dynamical matrix calculation in $\Gamma$ and six independent calculations of strain perturbation, which takes about 800 CPU hours. This makes a total walltime of about 2200 CPU hours.

In this particular case, the ratio is of about 18.5 in favor of the DFPT. For higher-symmetry systems, this ratio is less impressive, because of fewer relaxations in FD. However, for the lower-symmetry ones this ratio becomes much more important.

\section{SUMMARY AND CONCLUSIONS}

This study addresses the theoretical development and the implementation of the response to strain and electric field within the PAW approach of the DFPT. This implementation combines the numerical stability and the ease of use of the DFPT with the high precision and the speed of execution of the PAW formalism. This paper follows those of Audouze et al. [11] who developed, for the first time, the PAW approach in DFPT for the calculation of vibrational properties in ABINIT, and of Hamann et al. [3] who implemented the for-

TABLE V. Relaxed-ion elastic tensors of $\mathrm{Fe}_{16} \mathrm{C}$ (in GPa) at 320 GPa. The comparison between the perturbative approach (DFPT) and the finite difference approach (FD) shows differences on the order of tens of MPa or less.

\begin{tabular}{lccccl}
\hline \hline & $C_{11}$ & $C_{22}$ & $C_{33}$ & $C_{44}$ & $C_{55}$ \\
\hline FD & 127.409 & 127.444 & 47.284 & 25.254 & 25.244 \\
DFPT & 127.428 & 127.428 & 47.295 & 25.244 & 25.244 \\
& $C_{66}$ & $C_{12}$ & $C_{13}$ & $C_{14}$ & $C_{56}$ \\
FD & 41.037 & 45.339 & 31.510 & -2.739 & -2.739 \\
DFPT & 41.038 & 45.351 & 31.522 & -2.739 & -2.739 \\
\hline \hline
\end{tabular}


TABLE VI. Elastic constants (in GPa) and sound velocities (in $\mathrm{km} / \mathrm{s}$ ) of $\mathrm{MgSiO}_{3}$ perovskite at $0 \mathrm{GPa}$.

\begin{tabular}{lcccccc}
\hline \hline$C_{i j}$ & $C_{11}$ & $C_{22}$ & $C_{33}$ & $C_{44}$ & $C_{55}$ & \\
\hline DFPT+NC & 512 & 579 & 488 & 213 & 181 & \\
FD+PAW & 482 & 549 & 457 & 201 & 176 & \\
DFPT+PAW & 482 & 549 & 457 & 201 & 176 & \\
S.V. Sinogeikin (exp.)[31] & 481 & 528 & 456 & 200 & 182 & \\
Y.Haeri (exp.)[32] & 482 & 537 & 485 & 186 & 186 & \\
$C_{i j}$ & $C_{66}$ & $C_{13}$ & $C_{32}$ & $C_{12}$ & $V_{p}$ & $V_{s}$ \\
DFPT+NC & 166 & 153 & 167 & 162 & 11.21 & 6.63 \\
FD+PAW & 156 & 137 & 150 & 137 & 10.85 & 6.49 \\
DFPT+PAW & 156 & 137 & 150 & 137 & 10.85 & 6.49 \\
S.V. Sinogeikin (exp.)[31] & 147 & 139 & 146 & 125 & 10.84 & 6.47 \\
Y.Haeri (exp.) [32] & 147 & 147 & 146 & 144 & 11.04 & 6.57 \\
\hline \hline
\end{tabular}

mulation of the metric tensor and the calculation of the elastic properties in DFPT for norm-conserving pseudopotentials.

As in Hamann et al. [3], the central idea of our study is the development of the derivatives in terms of the metric tensor. The coupling of the strain perturbation with the electric field perturbation yields the piezoelectric tensors; the coupling with the atomic displacements provides the clamped and relaxed tensors. Because the PAW specificities, the formulation becomes more complicated than in the norm-conserving case.

Numerical tests show that the current implementation is robust and highly comparable to the standard stress-strain FD approach. An important aspect is to ensure a thorough k-point sampling of the Brillouin zone, because of the coupling with the electric field perturbations. However, the convergence is achieved faster than in the FD case.

The current implementation allows fast and accurate calculations of the elastic and related tensors even for large systems with low symmetry, as shown by initial test calculations on materials of geophysical interest with realistic compositions. Numerical comparisons on perovskite and iron supercells with impurities show that the ABINIT code can now be used on such systems containing a few tens of atoms. DFPT calculations are faster than conventional FD ones. It will therefore be possible to compute large cells, necessary to achieve low dilution rates.

\section{ACKNOWLEDGMENTS}

This research was supported in part by the European Research Council (ERC) under the European Union's Horizon 2020 research and innovation program (Grant Agreement No. 681818 IMPACT to R.C.). We acknowledge access to the GENCI supercomputers (Occigen, Ada, and Curie) through the st12816 series of eDARI computing grants.

\section{APPENDIX A: DERIVATIVES OF THE NON-LOCAL PROJECTORS IN A PLANE WAVE BASIS}

\section{Nonlocal energy}

The contribution of the non-local operator to the energy is

$$
E_{\mathrm{nl}}=\sum_{n} \sum_{\kappa, i j}\left\langle\tilde{\psi}_{n}^{(0)} \mid \tilde{p}_{i}^{\kappa}\right\rangle\left(D_{i j}^{\kappa}-\epsilon_{n} s_{i j}^{\kappa}\right)\left\langle\tilde{p}_{j}^{\kappa} \mid \tilde{\psi}_{n}^{(0)}\right\rangle,
$$

In this section, $i$ and $j$ indexes do not include the atom index $\kappa$ and run only over $l, m, n$ angular momenta.

The nonlocal projectors are expressed in terms of spherical harmonics:

$$
\tilde{p}_{i}^{\kappa}(\mathbf{r})=\frac{\bar{p}_{l_{i} n_{i}}(r)}{r} Y_{l_{i} m_{i}} \hat{(r)} .
$$

For the sake of brevity, we introduce the following notations:

$$
\begin{gathered}
V_{n, i j}^{\kappa}=D_{i j}^{\kappa}-\epsilon_{n} s_{i j}^{\kappa}, \\
\chi_{n, i}^{\kappa}=\left\langle\tilde{p}_{i}^{\kappa} \mid \tilde{\psi}_{n}^{(0)}\right\rangle .
\end{gathered}
$$

Note that in the usual case (collinear magnetism), we have $V_{n, i j}^{\kappa}=V_{n, j i}^{\kappa}$. Using these notations, the nonlocal energy is rewritten:

$$
E_{\mathrm{nl}}=\sum_{n} \sum_{\kappa, i j} V_{n, i j}^{\kappa} \chi_{n, i}^{\kappa *} \chi_{n, j}^{\kappa}=2 \sum_{n} \sum_{\kappa, i j} V_{n, i j}^{\kappa} \Re\left[\chi_{n, i}^{\kappa}{ }^{*} \chi_{n, j}^{\kappa}\right]
$$

The generalization to the non-collinear magnetism can be achieved using the hermiticity of $V_{n, i j}^{\kappa}$ scalars.

In this Appendix, we consider only the contributions to the nonlocal energy from the derivatives of the projectors. The wave functions are frozen. The contributions from $V_{n, i j}^{\kappa}$ derivatives have been detailed in Secs. III A 4 and III B 3. In these conditions, we consider the following partial derivatives of the nonlocal energy ( $\lambda_{i}$ runs over $\tau_{\kappa k}, \alpha \beta$ and $k_{j}$ ):

$$
\begin{aligned}
\frac{\partial E_{\mathrm{nl}}}{\partial \lambda_{1}} & =\sum_{n} \sum_{\kappa, i j} V_{n, i j}^{\kappa}\left\langle\tilde{\psi}_{n}^{(0)}\left|\frac{\partial\left|\tilde{p}_{i}^{\kappa}\right\rangle\left\langle\tilde{p}_{j}^{\kappa}\right|}{\partial \lambda_{1}}\right| \tilde{\psi}_{n}^{(0)}\right\rangle, \\
\frac{\partial^{2} E_{\mathrm{nl}}}{\partial \lambda_{1} \partial \lambda_{2}} & =\sum_{n} \sum_{\kappa, i j} V_{n, i j}^{\kappa}\left\langle\tilde{\psi}_{n}^{(0)}\left|\frac{\partial\left|\tilde{p}_{i}^{\kappa}\right\rangle\left\langle\tilde{p}_{j}^{\kappa}\right|}{\partial \lambda_{1} \partial \lambda_{2}}\right| \tilde{\psi}_{n}^{(0)}\right\rangle .
\end{aligned}
$$

They involve the partial derivatives of $\chi_{n, i}^{\kappa}$ factors:

$$
\begin{gathered}
\frac{\partial \chi_{n, i}^{\kappa}}{\partial \lambda_{1}}=\left\langle\frac{\partial \tilde{p}_{i}^{\kappa}}{\partial \lambda_{1}} \mid \tilde{\psi}_{n}^{(0)}\right\rangle, \\
\frac{\partial^{2} \chi_{n, i}^{\kappa}}{\partial \lambda_{1} \partial \lambda_{2}}=\left\langle\frac{\partial^{2} \tilde{p}_{i}^{\kappa}}{\partial \lambda_{1} \partial \lambda_{2}} \mid \tilde{\psi}_{n}^{(0)}\right\rangle .
\end{gathered}
$$

\section{Nonlocal form factors and their derivatives}

On a plane-wave basis, the $\chi_{n, i}^{\kappa}$ factors are developed as follows:

$$
\chi_{n, i}^{\kappa}=\frac{4 \pi}{\sqrt{\Omega}} i^{l_{i}} \sum_{\mathbf{G}} C_{\mathbf{G}}^{n} e^{2 \pi i \mathbf{K} \cdot \boldsymbol{\tau}_{\kappa}} F_{l_{i} m_{i} n_{i}}^{\kappa}(\mathbf{K}) .
$$

Where the $F_{l_{i} m_{i} n_{i}}^{\kappa}(\mathbf{K})$ are named nonlocal form factors, $C_{\mathbf{G}}^{n}=\left\langle\mathbf{G} \mid \tilde{\psi}_{n}^{(0)}\right\rangle$ and $\mathbf{K}=\mathbf{k}+\mathbf{G}$.

The nonlocal form factors have a radial and an angular part defined as

$$
F_{l m n}^{\kappa}(\mathbf{K})=Y_{l m}(\widehat{\mathbf{K}}) f_{n l}^{\kappa}(K)
$$

with

$$
f_{n l}^{\kappa}(K)=\int \bar{p}_{l n}^{\kappa}(r) j_{l}(K r) r d r .
$$

$j_{l}(x)$ are the first-order spherical Bessel functions. 
The first and second order derivatives of a non-local form factor with respect to the wave vector are expressed in terms of reduced coordinates:

$$
\begin{aligned}
\frac{\partial F_{l m n}^{\kappa}(\mathbf{K})}{\partial K_{\gamma}} & =\sum_{\mu} G_{\gamma}^{\mu} \tilde{F}_{l m n, \mu}^{\prime \kappa}(\mathbf{K}), \\
\frac{\partial^{2} F_{l m n}^{\kappa}(\mathbf{K})}{\partial K_{\gamma} \partial K_{\delta}} & =\sum_{\mu \nu} G_{\gamma}^{\mu} G_{\delta}^{\nu} \tilde{F}_{l m n, \mu \nu}^{\prime \prime}(\mathbf{K}) .
\end{aligned}
$$

The reduced components are straightforwardly obtained from

$$
\begin{aligned}
\tilde{F}_{l m n, \mu}^{\prime \kappa}(\mathbf{K})= & \left(\frac{\partial Y_{l m}(\widehat{\mathbf{K}})}{\partial \mathbf{K}}\right)_{\mu}^{\mathrm{red}} f_{n l}^{\kappa}(K)+\frac{f_{l l}^{\kappa^{\prime}}(K)}{K} Y_{l m}(\widehat{\mathbf{K}}) \tilde{K}_{\mu}, \\
\tilde{F}_{l m n, \mu \nu}^{\prime \prime}(\mathbf{K})= & \left(\frac{\partial^{2} Y_{l m}(\widehat{\mathbf{K}})}{\partial \mathbf{K} \partial \mathbf{K}}\right)_{\mu \nu}^{\mathrm{red}} f_{n l}^{\kappa}(K), \\
& +\tilde{K}_{\mu} \tilde{K}_{\nu} \frac{f_{n l}^{\kappa^{\prime \prime}}(K)}{K^{2}} Y_{l m}(\widehat{\mathbf{K}})+\left[\left(\Xi_{\mu \nu}-\frac{\tilde{K}_{\mu} \tilde{K}_{v}}{K^{2}}\right) Y_{l m}(\widehat{\mathbf{K}}),\right. \\
& \left.+\left(\frac{\partial Y_{l m}(\widehat{\mathbf{K}})}{\partial \mathbf{K}}\right)_{\mu}^{\mathrm{red}} \tilde{K}_{v}+\left(\frac{\partial Y_{l m}(\widehat{\mathbf{K}})}{\partial \mathbf{K}}\right)_{\nu}^{\mathrm{red}} \tilde{K}_{\mu}\right] \frac{f_{l l}^{\kappa^{\prime}}(K)}{K},
\end{aligned}
$$

where

$$
\begin{gathered}
\left(\frac{\partial Y_{l m}(\widehat{\mathbf{K}})}{\partial \mathbf{K}}\right)_{\mu}^{\mathrm{red}}=\sum_{\alpha} R_{\mu}^{\alpha} \frac{\partial Y_{l m}(\widehat{\mathbf{K}})}{\partial K_{\gamma}} \\
\left(\frac{\partial^{2} Y_{l m}(\widehat{\mathbf{K}})}{\partial \mathbf{K} \partial \mathbf{K}}\right)_{\mu \nu}^{\mathrm{red}}=\sum_{\mu \nu} R_{\nu}^{\gamma} R_{\mu}^{\delta} \frac{\partial Y_{l m}(\widehat{\mathbf{K}})}{\partial K_{\gamma} K_{\delta}}
\end{gathered}
$$

$f_{n l}^{\kappa^{\prime}}$ and $f_{n l}^{\kappa^{\prime \prime}}$ are the first and second order derivatives of the non-local radial function $f_{n l}^{\kappa}$.

The first-order derivative of a non-local form factor with respect to the deformation $\varepsilon_{\alpha \beta}$ is obtained as follows:

$$
\begin{aligned}
\frac{\partial F_{l m n}^{\kappa}(\mathbf{K})}{\partial \varepsilon_{\alpha \beta}} & =\sum_{\gamma} \frac{\partial F_{l m n}^{\kappa}(\mathbf{K})}{\partial K_{\gamma}} \frac{\partial K_{\gamma}}{\partial \varepsilon_{\alpha \beta}} \\
& =\sum_{\gamma}\left(\sum_{\mu} \tilde{F}_{l m n, \mu}^{\prime \kappa}(\mathbf{K}) G_{\gamma}^{\mu}\right)\left(-\sum_{\nu} G_{\beta}^{v} \tilde{K}_{\nu} \delta_{\alpha \gamma}\right) \\
& =-\sum_{\mu \nu} G_{\alpha}^{\mu} G_{\beta}^{v} \tilde{F}_{l m n, \mu}^{\prime \kappa}(\mathbf{K}) \tilde{K}_{\nu}
\end{aligned}
$$

Using the same development, we also can deduce the expressions for the second order derivatives involving the deformation:

$$
\begin{aligned}
\frac{\partial^{2} F_{l m n}^{\kappa}(\mathbf{K})}{\partial \varepsilon_{\alpha \beta} \partial \varepsilon_{\gamma \delta}}=\sum_{\mu \nu \lambda \rho}\left[G _ { \alpha } ^ { \mu } G _ { \beta } ^ { \nu } G _ { \gamma } ^ { \lambda } G _ { \delta } ^ { \rho } \left(\tilde{F}_{l m n, \mu \lambda}^{\prime \prime}(\mathbf{K}) \tilde{K}_{\nu} \tilde{K}_{\rho}\right.\right. & \left.\left.+\Xi_{\mu \rho} \tilde{F}_{l m n, \mu}^{\prime \kappa}(\mathbf{K}) \tilde{K}_{\nu}\right)\right] \\
\frac{\partial^{2} F_{l m n}^{\kappa}(\mathbf{K})}{\partial \varepsilon_{\alpha \beta} \partial K_{\gamma}}= & \sum_{\mu \nu \lambda} \Upsilon_{\gamma \mu} G_{\alpha}^{\mu} G_{\beta}^{\lambda} \tilde{F}_{l m n, \mu \nu}^{\prime \prime}(\mathbf{K}) \tilde{K}_{\lambda} \\
& -G_{\beta}^{\gamma} \sum_{\mu} G_{\alpha}^{\mu} \tilde{F}_{l m n, \mu}^{\prime \kappa}(\mathbf{K})
\end{aligned}
$$

\section{Contribution to the stress tensor}

$$
\sigma_{\alpha \beta}^{\mathrm{nl}}=\frac{1}{\Omega} \frac{\partial E_{\mathrm{nl}}}{\partial \varepsilon_{\alpha \beta}}=\frac{2}{\Omega} \sum_{n} \sum_{\kappa, i j} V_{n, i j}^{\kappa} \Re\left[\frac{\partial \chi_{n, i}^{\kappa}{ }^{*}}{\partial \varepsilon_{\alpha \beta}} \chi_{n, j}^{\kappa}\right]
$$

with

$$
\begin{aligned}
\frac{\partial \chi_{n, i}^{\kappa}}{\partial \varepsilon_{\alpha \beta}}= & \frac{4 \pi}{\sqrt{\Omega}} i^{l_{i}} \sum_{\mathbf{G}} C_{\mathbf{G}}^{n} e^{2 \pi i \mathbf{K} \cdot \boldsymbol{\tau}_{\kappa}} \frac{\partial F_{l m n}^{\kappa}(\mathbf{K})}{\partial \varepsilon_{\alpha \beta}} \\
& -\frac{\delta_{\alpha \beta}}{2} \chi_{n, i}^{\kappa}
\end{aligned}
$$

\section{Contribution to the elastic tensor}

$$
\begin{aligned}
C_{\alpha \beta \gamma \delta}^{\mathrm{nl}} & =\frac{1}{\Omega} \frac{\partial^{2} E_{\mathrm{nl}}}{\partial \varepsilon_{\alpha \beta} \partial \varepsilon_{\gamma \delta}} \\
& =\frac{2}{\Omega} \sum_{n} \sum_{\kappa, i j} V_{n, i j}^{\kappa} \Re\left[\frac{\partial^{2} \chi_{n, i}^{\kappa}}{\partial \varepsilon_{\alpha \beta} \partial \varepsilon_{\gamma \delta}} \chi_{n, j}^{\kappa}+\frac{\partial \chi_{n, i}^{\kappa}{ }^{*}}{\partial \varepsilon_{\alpha \beta}} \frac{\partial \chi_{n, j}^{\kappa}}{\partial \varepsilon_{\gamma \delta}}\right]
\end{aligned}
$$

with

$$
\begin{aligned}
\frac{\partial^{2} \chi_{n, i}^{\kappa}}{\partial \varepsilon_{\alpha \beta} \partial \varepsilon_{\gamma \delta}}= & \frac{4 \pi}{\sqrt{\Omega}} i^{l_{i}} \sum_{\mathbf{G}} C_{\mathbf{G}}^{n} e^{2 \pi i \mathbf{K} \cdot \boldsymbol{\tau}_{\kappa}} \frac{\partial^{2} F_{l m n}^{\kappa}(\mathbf{K})}{\partial \varepsilon_{\alpha \beta} \partial \varepsilon_{\gamma \delta}} \\
& -\frac{\delta_{\gamma \delta}}{2} \frac{\partial \chi_{n, i}^{\kappa}}{\partial \varepsilon_{\alpha \beta}}-\frac{\delta_{\alpha \beta}}{2} \frac{\partial \chi_{n, i}^{\kappa}}{\partial \varepsilon_{\gamma \delta}}
\end{aligned}
$$

\section{Contribution to the force-strain coupling tensor}

$$
\begin{aligned}
\Lambda_{\kappa k \alpha \beta}^{\mathrm{nl}} & =-\frac{\partial^{2} E_{\mathrm{nl}}}{\partial \tau_{\kappa k} \partial \varepsilon_{\alpha \beta}} \\
& =-2 \sum_{n} \sum_{\kappa, i j} V_{n, i j}^{\kappa} \Re\left[\frac{\partial^{2} \chi_{n, i}^{\kappa *}}{\partial \varepsilon_{\alpha \beta} \partial \tau_{\kappa k}} \chi_{n, j}^{\kappa}+\frac{\partial \chi_{n, i}^{\kappa}}{\partial \varepsilon_{\alpha \beta}} \frac{\partial \chi_{n, j}^{\kappa}}{\partial \tau_{\kappa k}}\right]
\end{aligned}
$$

with

$$
\begin{aligned}
\frac{\partial \chi_{n, i}^{\kappa}}{\partial \tau_{\kappa k}}= & \frac{4 \pi}{\sqrt{\Omega}} i^{l_{i}} \sum_{\mathbf{G}} C_{\mathbf{G}}^{n} \tilde{K}_{k} e^{2 \pi i \mathbf{K} \cdot \boldsymbol{\tau}_{\kappa}} F_{l m n}^{\kappa}(\mathbf{K}) \\
\frac{\partial^{2} \chi_{n, i}^{\kappa}}{\partial \varepsilon_{\alpha \beta} \partial \tau_{\kappa k}}= & \frac{4 \pi}{\sqrt{\Omega}} i^{l_{i}} \sum_{\mathbf{G}} C_{\mathbf{G}}^{n} \tilde{K}_{k} e^{2 \pi i \mathbf{K} \cdot \tau_{\kappa}} \frac{\partial F_{l m n}^{\kappa}(\mathbf{K})}{\partial \varepsilon_{\alpha \beta}} \\
& -\frac{\delta_{\alpha \beta}}{2} \frac{\partial \chi_{n, i}^{\kappa}}{\partial \tau_{\kappa k}}
\end{aligned}
$$

\section{Contribution to the piezoelectric tensor}

To compute $e_{\gamma \alpha \beta}^{\mathrm{nl}}=-\frac{1}{\Omega} \frac{\partial^{2} E_{\mathrm{nl}}}{\partial \mathcal{E}_{\gamma} \partial \varepsilon_{\alpha \beta}}$, we need (see Eq. 90):

$$
\begin{aligned}
& \sum_{n} \sum_{\kappa, i j} V_{n, i j}^{\kappa}\left|\tilde{\psi}_{n}^{(0)}\right| \frac{\partial}{\partial \varepsilon_{\alpha \beta}}\left(\left|\tilde{p}_{i}^{\kappa}\right\rangle \frac{\partial\left\langle\tilde{p}_{j}^{\kappa}\right|}{\partial K_{\gamma}}\right)\left|\tilde{\psi}_{n}^{(0)}\right\rangle \\
& =\sum_{n} \sum_{\kappa, i j} V_{n, i j}^{\kappa}\left[\frac{\partial^{2} \chi_{n, i}^{\kappa}{ }^{*}}{\partial \varepsilon_{\alpha \beta} \partial K_{\gamma}} \chi_{n, j}^{\kappa}+\frac{\partial \chi_{n, i}^{\kappa}{ }^{*}}{\partial \varepsilon_{\alpha \beta}} \frac{\partial \chi_{n, j}^{\kappa}}{\partial K_{\gamma}}\right],
\end{aligned}
$$


with

$$
\begin{aligned}
\frac{\partial^{2} \chi_{n, i}^{\kappa}}{\partial \varepsilon_{\alpha \beta} \partial \tilde{K}_{\gamma}}= & \frac{4 \pi}{\sqrt{\Omega}} i^{l_{i}} \sum_{\mathbf{G}} C_{\mathbf{G}}^{n} e^{2 \pi i \mathbf{K} \cdot \boldsymbol{\tau}_{\kappa}} \frac{\partial^{2} F_{l m n}^{\kappa}(\mathbf{K})}{\partial \varepsilon_{\alpha \beta} \partial K_{\gamma}} \\
& -\frac{\delta_{\alpha \beta}}{2} \frac{\partial \chi_{n, i}^{\kappa}}{\partial K_{\gamma}}
\end{aligned}
$$

\section{Contribution to the effective charges}

To compute $Z_{\kappa k \gamma}^{\mathrm{nl}}=-\frac{\partial^{2} E_{\mathrm{nl}}}{\partial \mathcal{E}_{\gamma} \partial \tau_{k k}}$, we need [see Eq. (90)]:

$$
\begin{aligned}
& \sum_{n} \sum_{\kappa, i j} V_{n, i j}^{\kappa}\left|\tilde{\psi}_{n}^{(0)}\right| \frac{\partial}{\partial \tau_{\kappa k}}\left(\left|\tilde{p}_{i}^{\kappa}\right\rangle \frac{\partial\left\langle\tilde{p}_{j}^{\kappa}\right|}{\partial K_{\gamma}}\right)\left|\tilde{\psi}_{n}^{(0)}\right\rangle \\
& =\sum_{n} \sum_{\kappa, i j} V_{n, i j}^{\kappa}\left[\frac{\partial^{2} \chi_{n, i}^{\kappa}{ }^{*}}{\partial \tau_{\kappa k} \partial K_{\gamma}} \chi_{n, j}^{\kappa}+\frac{\partial \chi_{n, i}^{\kappa}{ }^{*}}{\partial \tau_{\kappa k}} \frac{\partial \chi_{n, j}^{\kappa}}{\partial K_{\gamma}}\right],
\end{aligned}
$$

with

$$
\frac{\partial^{2} \chi_{n, i}^{\kappa}}{\partial \tau_{\kappa k} \partial K_{\gamma}}=\frac{4 \pi}{\sqrt{\Omega}} i^{l_{i}} \sum_{\mathbf{G}} C_{\mathbf{G}}^{n} \tilde{K}_{k} e^{2 \pi i \mathbf{K} \cdot \tau_{\kappa}} \frac{\partial F_{l m n}^{\kappa}(\mathbf{K})}{\partial K_{\gamma}} .
$$

\section{APPENDIX B: LIMITS AT ZERO OF THE RADIAL FOURIER TRANSFORM OF THE ATOMIC PSEUDO-CORE-DENSITY}

To compute the nonlinear core correction to exchange and correlation first-order potential, we need the limit at zero of the radial Fourier transform of the atomic pseudo-core-density derivatives $\tilde{\rho}_{c}^{\kappa}(G)$ [see Eqs. (58) and (74)]. These limits are straightforwardly obtained by developing the Sinc function near zero:

$$
\begin{gathered}
\tilde{\rho}_{c}^{\kappa}(G)=\frac{4 \pi}{\Omega} \int_{0}^{\infty} \tilde{\rho}_{c}^{\kappa}(r) r^{2} \frac{\sin (G r)}{G r} d r, \\
\lim _{G \rightarrow 0} \tilde{\rho}_{c}^{\kappa}(G)=\frac{4 \pi}{\Omega} \int_{0}^{\infty} \tilde{\rho}_{c}^{\kappa}(r) r^{2} d r, \\
\lim _{G \rightarrow 0} \frac{\tilde{\rho}_{c}^{\kappa}(G)}{G}=-\frac{1}{3} \frac{4 \pi}{\Omega} \int_{0}^{\infty} \tilde{\rho}_{c}^{\kappa}(r) r^{4} d r, \\
\lim _{G \rightarrow 0}\left(\frac{\tilde{\rho}_{c}^{\kappa \prime}(G)}{G^{2}}-\frac{\tilde{\rho}_{c}^{\kappa \prime}(G)}{G^{3}}\right)=\frac{29}{30} \frac{4 \pi}{\Omega} \int_{0}^{\infty} \tilde{\rho}_{c}^{\kappa}(r) r^{6} d r .
\end{gathered}
$$

[12] X. Gonze and J.-P. Vigneron, Phys. Rev. B 39, 13120 (1989).

[13] X. Gonze, Phys. Rev. A 52, 1086 (1995).

[14] X. Gonze, Phys. Rev. A 52, 1096 (1995).

[15] X. Gonze and C. Lee, Phys. Rev. B 55, 10355 (1997).

[16] C. Audouze, F. Jollet, M. Torrent, and X. Gonze, Phys. Rev. B 78, 035105 (2008).

[17] X. Wu, D. Vanderbilt, and D. R. Hamann, Phys. Rev. B 72, 035105 (2005).

[18] X. Gonze, Phys. Rev. B 55, 10337 (1997).

[19] P. Umari, X. Gonze, and A. Pasquarello, Phys. Rev. B 69, 235102 (2004).

[20] K. Miwa, Phys. Rev. B 84, 094304 (2011).

[21] S. G. Louie, S. Froyen, and M. L. Cohen, Phys. Rev. B 26, 1738 (1982).

[22] A. DalCorso, Phys. Rev. B 64, 235118 (2001).

[23] S. Baroni, S. de Gironcoli, A. Dal Corso, and P. Giannozzi, Rev. Mod. Phys. 73, 515 (2001).

[24] X. Gonze, B. Amadon, P.-M. Anglade, J.-M. Beuken, F. Bottin, P. Boulanger, F. Bruneval, D. Caliste, R. Caracas, M. Côté, T. Deutsch, L. Genovese, P. Ghosez, M. Giantomassi, S. Goedecker, D. Hamann, P. Hermet, F. Jollet, G. Jomard, S. Leroux, M. Mancini, S. Mazevet, M. Oliveira, G. Onida, Y. Pouillon, T. Rangel, G.-M. Rignanese, D. Sangalli, R. Shaltaf, M. Torrent, M. Verstraete, G. Zerah, and J. Zwanziger, Comput. Phys. Commun. 180, 2582 (2009).

[25] X. Gonze, G.-M. Rignanese, M. Verstraete, J.-M. Beuken, Y. Pouillon, R. Caracas, F. Jollet, M. Torrent, G. Zerah, M. Mikami, P. Ghosez, M. Veithen, J.-Y. Raty, V. Olevano, F. Bruneval, L. Reining, R. Godby, G. Onida, D. Hamann, and D. Allan., Z. Kristallogr. 220, 558 (2004).

[26] X. Gonze, J.-M. Beuken, R. Caracas, F. Detraux, M. Fuchs, G.-M. Rignanese, L. Sindic, M. Verstraete, G. Zerah, F. Jollet, M. Torrent, A. Roy, M. Mikami, P. Ghosez, J.-Y. Raty, and D. Allan, Comput. Mater. Sci. 25, 478 (2002). 
[27] R. Caracas and R. E. Cohen, Effect of Chemistry on the Physical properties of perovskite and post-perovskite, in PostPerovskite: The Last Mantle Phase Transition, edited by K. Hirose, J. Brodholt, T. Lay, and D. Yuen (American Geophysical Union, 2007), Vol. 174, pp. 115-128.

[28] S. de Gironcoli, Phys. Rev. B 51, 6773 (1995).
[29] G. N. Kamm and G. A. Alers, J. Appl. Phys. 35, 327 (1964).

[30] R. Caracas, Geophys. Res. Lett. 44, 128 (2017).

[31] S. V. Sinogeikin, J. Zhang, and J. D. Bass, Geophys. Res. Lett. 31, L06620 (2004).

[32] A. Yeganeh-Haeri, Phys. Earth Planet. 87, 111 (1971). 